\title{
Der référé législatif- österreichische Gesetzgebungsgeschichte zwischen Rechtsvereinheitlichung und Justizsteuerung
}

\begin{abstract}
As judicial submission requirement to a legislative commission the référé législatif incapacitates judges as mechanical legal practitioners under the tutelage of the monarchical legislatif will. It can be found in the Austrian legislative history in various provisions reaching from voluntary consulting in the "Landesordnung" and in the "Codex Theresianus" to a strict submission duty in the "Allgemeine Gerichtsordnung" and especially in the "Josephinisches Gesetzbuch". In the "Patent of 1791" the judicial autonomy of legal interpretation was restored, though it was in the "Westgalizisches Gesetzbuch" that the référé légilatif was completely abolished. This paper shows that the introduction of the référé législatif aims at multiple objectives. So far, research concentrates on the objective of enforcement of the legislator's intentions and doesn't consider the reputation of the judge underlying the introdcution of the submission requirement. Looking onto the legislative materials each judicial submission requirement is a distrust statement againt the judiciary, that has to be controlled. This controlling function of the référé législatif was exploited by the conflict between monarchic centralism and the estates`rights of self-administration.
\end{abstract}

\section{Einleitung: \\ Definition des Gegenstandes und Forschungsstand}

Nach den Art. $87^{1}$ und $88^{2}$ B-VG i.d.F. v. 14. Dezember $2010^{3}$ ist ein österreichischer Rich-

\footnotetext{
${ }^{1}$ Art. 87. (1) Die Richter sind in Ausübung ihres richterlichen Amtes unabhängig. (2) In Ausübung seines richterlichen Amtes befindet sich ein Richter bei Besorgung aller ihm nach dem Gesetz und der Geschäftsverteilung zustehenden gerichtlichen Geschäfte, mit Ausschluss der Justizverwaltungssachen, die nicht nach Vorschrift des Gesetzes durch Senate oder Kommissionen zu erledigen sind. (3) Die Geschäfte sind unter die Richter eines Gerichtes für die in der Gerichtsverfassung bestimmte Zeit im Voraus zu verteilen. Eine nach dieser Geschäftsverteilung einem Richter zufallende Sache darf ihm nur durch Verfügung des durch die Gerichtsverfassung hierzu berufenen Senates und nur im Fall seiner Verhinderung oder dann abgenommen werden, wenn er wegen des
}

Umfangs seiner Aufgaben an deren Erledigung innerhalb einer angemessenen Frist gehindert ist.

${ }^{2}$ Art. 88. (1) Durch Bundesgesetz wird eine Altersgrenze bestimmt, mit deren Erreichung die Richter in den dauernden Ruhestand treten. (2) Im Übrigen dürfen Richter nur in den vom Gesetz vorgeschriebenen Fällen und Formen und auf Grund eines förmlichen richterlichen Erkenntnisses ihres Amtes entsetzt oder wider ihren Willen an eine andere Stelle oder in den Ruhestand versetzt werden. Diese Bestimmungen finden jedoch auf Übersetzungen und Versetzungen in den Ruhestand keine Anwendung, die durch Veränderungen in der Verfassung der Gerichte nötig werden. In einem solchen Fall wird durch das Gesetz festgestellt, innerhalb welchen Zeitraumes Richter ohne die sonst vorgeschriebenen Förmlichkeiten übersetzt und in den Ruhestand versetzt werden können. (3) Die zeitweise Enthebung der Richter vom Amt darf nur durch Verfügung des Gerichtsvorstandes oder der höheren Gerichtsbehörde bei gleichzeitiger Verweisung der Sache an das zuständige Gericht stattfinden. 
ter bei der Rechtsfindung und Rechtsprechung als unabhängiges Staatsorgan tätig. Ein Wesensmerkmal der richterlichen Unabhängigkeit ist die Weisungsungebundenheit des Richters, also seine sachliche Unabhängigkeit. Diese garantiert nach den einschlägigen Verfassungskommentaren, dass der Richter nur an das Gesetz gebunden ist und nach seiner eigenen Rechtsüberzeugung entscheidet. Hier liegt der Anknüpfungspunkt für meine Überlegungen zum référé législatif. Der französische Begriff "référé législatif" ${ }^{4}$ meint die richterliche Vorlage(pflicht) an den Gesetzgeber. Damit soll der Richter als mechanischer Rechtsanwender auf den Willen des (monarchischen) Gesetzgebers verpflichtet werden. Die Spannungslage zur richterlichen Unabhängigkeit ist offensichtlich. Der référé législatif ist Ventil des monarchischen Absolutheitsanspruchs, der in Gesetzgebung und Gerichtsbarkeit Hoheitsrechte des Monarchen sieht. ${ }^{5}$ Die Durchsetzung monarchischer Justizhoheit mittels Vorlagepflicht ist eingebettet in das Bestreben, ständisch beeinflusste Spruchkörper zugunsten landesherrlicher Zentralbehörden zurückzudrängen. Daher der eine Teil des Aufsatztitels: Justizkontrolle.

\footnotetext{
${ }^{3}$ Abschnitt Gerichtsbarkeit - nach den Universitäten.

${ }^{4}$ Siehe zur Entwicklung des Begriffs in Frankreich seit Beginn des 19. Jahrhunderts MERLIN, Répertoire universel 695: „RÉFÉRÉ AU LÉGISLATEUR. On appelait ainsi autrefois un jugement par lequel, avant de prononcer sur une question qui leur paraissait insoluble, d'après l'ambiguité ou l'insuffisance de la loi dont elle dérivait, les juges ordonnaient qu'il en serait référé à l'autorité investie du pouvoir législatif". - In den Wörterbüchern Grand Larousse de la langue française und Le Grand Robert de la langue française wird der référé législatif als "[...] demande faite par le juge au législateur en vue d'interpréter une loi" bzw. "demande d'interprétation de la loi, adressée par un juge au législateur" bezeichnet. Für die französische Definition siehe außerdem HufTEAU, Référé législatif 1 u. 9; GENY, Méthode d'interprétation 78 .

${ }^{5}$ SEIF, Absolutismus 30-38.
}

Hinter der Verpflichtung des Richters auf den Gesetzgeberwillen steht aber auch der Rationalitätsanspruch des Reformabsolutismus. Kodifikationen setzen die aufgeklärt absolutistische Staatsräson durch, die den Fürstenstaat als gesetzmäßiges Herrschaftsinstrument neu ordnet und dem Richter keine Entscheidungsfreiheit zugestehen will. ${ }^{6}$ - Daher der andere Teil des Aufsatztitels: Rechtsvereinheitlichung.

Die entscheidenden Weichenstellungen für den référé législatif in der österreichischen Rechtsgeschichte finden sich im Kodifikationsprozess vom Codex Theresianus bis hin zum Urentwurf des Westgalizischen Gesetzbuches. Das 17. Jahrhundert wird hier am Rande miteinbezogen, da sich sowohl unter Ferdinand II. als auch unter Ferdinand III. Quellenbelege finden, die die richterliche Entscheidungsfreiheit beschränken. Der Forschungsstand findet sich in den grundlegenden Arbeiten von Ludwig Spiegel und Thomas Simon. Die Rekonstruktion der Gesetzesberatungen gelingt trotz Verlustes eines Großteils des Quellenbestandes anhand der grundlegenden Darstellungen von v. Harrasowsky $^{7}$ und Ofner. ${ }^{8}$

Untersuchungsmaßstab ist nach der eingangs skizzierten Definition des référé législatif die Verpflichtung des Richters, bei einer Gesetzeslücke oder einem Zweifel über Inhalt oder Anwendbarkeit eines Gesetzes das anhängige Verfahren auszusetzen, um die Entscheidung der Zweifelsfrage dem Monarchen oder einem vom Monar-

\footnotetext{
${ }^{6}$ SIMON, Gesetzgebungsstaat 70.

${ }^{7}$ HarrasowsKY, Geschichte der Codification; DERS., Codex Theresianus.

${ }^{8}$ OfNER, Ur-Entwurf 11: Nach dem Vortrag des Referenten v. Zeillers bildeten diese 17 Kommissionen: das niederösterreichische, innerösterreichische, oberösterreichische, böhmische, mährisch-schlesische und ostgalizische Appellationsgericht, dann die Landrechte in Österreich ob der Enns, Steiermark, Krain, Görz, Triest, zu Stanislau und Tarnow und die Fakultäten in Wien, Prag, Innsbruck und Freiburg.
} 
chen betrauten Regierungsorgan vorzulegen. ${ }^{9}$ Dadurch war die Quellenlektüre nicht nur auf die richterliche Vorlage- und Anfragepflicht im engeren Sinn fokussiert, sondern bezog auch Formen der authentischen Interpretation ein. ${ }^{10}$

\section{Entstehungsgeschichte des référé législatif in den österreichischen Quellen}

\section{Der référé législatif im 17. Jahrhundert}

Noch bevor das Richterleitbild eines mechanischen Rechtsanwenders vernunftrechtlicher Kodifikationen am Horizont der Geschichte erkennbar ist, findet sich in der verneuerten Landesordnung für Böhmen vom 10. Mai 162711

\footnotetext{
${ }^{9}$ Dazu LuKAS, Zur Lehre 407; BECKER, Kommentierund Auslegungsverbote 967; CARONI, Kodifikation 915; CONRAD, Deutsche Rechtsgeschichte 2, 385; VOGEL, Zur Praxis und Theorie der richterlichen Bindung 20; SPIEGEL, Référé législatif 100; IMMEL, Typologie der Gesetzgebung 93.

${ }^{10}$ Mohnhaupt, Potestas legislatoria 223, der von fließenden Übergängen zwischen der authentischen Interpretation und dem référé législatif ausgeht. Vgl. auch DROSTE-LEHNEN, Die authentische Interpretation 238f. $\mathrm{Zu}$ den Auffassungen über die authentische Interpretation vom Römischen Recht bis zu Beginn des 19. Jahrhunderts BERGMANN, Verbot 1, 60f.; 2, 176f., 196f., 322f. A.A. LUKAS, Zur Lehre 404f., der die beiden Begriffe trennt.

${ }^{11}$ Unter der Überschrift des Titels „Von Urteilen und Rechts-Sprüchen, so auf Erkäntnus der Sache gesprochen werden". Die verneuerte Landesordnung war das am 10. 5. 1627 durch Ferdinand II. erlassene absolutistische Grundgesetz für das Königreich Böhmen, das nach dem Scheitern des Aufstandes der protestantischen böhmischen Stände (2. Prager Fenstersturz 1618, Confoederatio Bohemica 1619, Schlacht am Weißen Berg 1620) die weitreichenden Machtbefugnisse der habsburgischen Krone festschrieb. Die grundgesetzliche Fixierung der Königsherrschaft in der "Verneuerten Landesordnung“ zeigt markante Entwicklungstendenzen des frühmodernen Fürsten-
}

in D 49 folgende einschlägige Regelung: „Wie nun im Versprechen [d.h. in Urteilen] auf diese Unsere Vernewerte Landesordnung zu gehen und, so etwas daraus allegiert, [...] und von denen Landrechtsbeisitzern nachzuschlagen ist [d.h. in einem Fall, der nicht in der Landesordnung ausdrücklich geregelt ist], [...] oder praeiudicata [d.h. Präjudizien] gesehen und fast die Urteil darauf gerichtet worden jedoch, weil solches sehr gefährlich und dadurch große Ungerechtigkeiten begangen werden können, auch dergleichen praeiudicata öfters einander selbst zuwider, zudem ihrer sehr wenigen bekannt sein: als haben Wir gnädigst anbefohlen, daß Unsere Land-Offizierer und LandrechtsBeisitzer, [...] die zweifelhaftigen Fälle, so bishero mehrern teils durch die Nàlez [tschechisch für freie Rechtsfindung] haben entschieden werden müssen und so in dieser Unserer Landesordnung sich nicht befinden, zusammentragen, dieselben in Ordnung bringen und nachmals neben ihrem Gutachten bei einem jeden Fall oder zweifelhaftigen Rechtsfrag Uns solches zu Unserer decision (welche darauf unfehlbar erfolgen wird) zu Handen Unserer Königlichen Böhmischen Hofkanzlei einantworten sollen. Inmittelst aber soll allein nach dieser Unserer Vernewerten Landesordnung, auch wann sich etwan ein Fall ereignete, so in der Landesordnung nicht erörtert, in dem Stadtrecht aber sich desselben Dezision befünde, nach dem Stadtrechten [...] judiziert und in diesen wie in allen anderen Rechten nicht allein auf die Wort, sondern auch die rationes legis gegangen werden." 12

\footnotetext{
staates, indem die Abkehr von der überkommenen "monarchia mixta" und die Durchsetzung der "potestas absoluta" des Fürsten bewusst angestrebtes Ziel der Verfassungsgebung ist. Die deutsche Sprache wurde neben dem Tschechischen Amtssprache. Vgl. ausführlich: BERGERHAUSEN, Die „Verneuerte Landesordnung" in Böhmen 1627 327-351.

12 Zit. n. SpIEGEL, Référé législatif 104.
} 
Wenn auch die Forschung vereinzelt mit KrainzEhrenzweig (in seinem System des österreichischen allgemeinen Privatrechts) diese Bestimmung Ferdinands II. dahingehend interpretiert, dass die Lücken der verneuerten Landesordnung für Böhmen zunächst aus dem Stadtrecht $\mathrm{zu}$ ergänzen waren und dann erst die landesfürstliche Entscheidung eingeholt worden ist, möchte ich mit Ludwig Spiegel darauf hinweisen, dass die königlichen Entscheidungen in die Zukunft gerichtet waren und keine Weisung für den Einzelfall beanspruchten. ${ }^{13}$ Nach den Rechtsprechungsbelegen der böhmischen Untergerichte orientierten sich die Richter auch nach 1627 an den "rationes legis" und waren nicht auf den Wortlaut fixiert. Dem Richter war die Entscheidungsfreiheit im jeweiligen Rechtsfall belassen ${ }^{14}$ und ein référé noch nicht gegeben. Schon wurde allerdings die "Gefährlichkeit widersprüchlicher praeiudicata" beschworen, und das Kundmachungspatent beanspruchte in Anlehnung an die justinianische Novelle $113^{15}$ das Recht des "ius legis ferendae" ausschließlich für den Monarchen. Gerade letzteres fällt im Unterschied zu den Landesordnungen von 1549 und 1564 auf, welche noch für Herren und Ritter das Recht formuliert haben, ihre Rechte mit Zu-

\footnotetext{
${ }^{13}$ Im Gerichtsgebrauch, der aus der freien richterlichen Rechtsschöpfung resultiert, und in den Präjudizien werden Gefahren erkannt, die sich in Ungerechtigkeiten und Widersprüchen ausdrücken. Zudem wird festgestellt, dass Präjudizien zu wenig bekannt seien, so dass die Notwendigkeit gesehen wird, den Gerichtsgebrauch und die Präjudizien zu ordnen, zu begutachten und höchsten Ortes über die weitere Anwendbarkeit $\mathrm{zu}$ entscheiden. Diese königlichen Dezisionen stellen dann Novellen der Landesordnung dar.

14 Siehe auch SpIEgel, Référé législatif 104, der unter Anm. 3 auch Verfasser erwähnt, die schon hier von dem Vorliegen eines référé législatif ausgehen; KRAINZ, EHRENZWEIG, System 26.

${ }^{15}$ SPIEGEL, Référé législatif 103f.; Novelle 113, in: OTTO, SCHILling, SINTENIS, Corpus Iuris Civilis 527f.
}

stimmung des Königs zu mehren und zu mindern. ${ }^{16}$

In einem kaiserlichen Reskript aus dem Jahre 1630 ist überliefert, „daß in casibus dubiis an Ihro Majestät um Belehrung zu rekurrieren“" sei. ${ }^{17} 1640$ formulierten die von Ferdinand II. erlassenen "Novellen“ und "Deklaratorien“18 unter C c 5 für Richter das Verbot der selbständigen Lückenausfüllung: „Nachdem in der verneuerten Landesordnung D 49 [...] nicht versehen, wohin in denen Fällen, welche auch in den Stadtrechten nicht begriffen, zu rekurrieren, und nun solches eine Sache ist, so billig seine Gewißheit haben soll: als setzen, ordnen und wollen Wir, daß man nicht wiederumb zu den ungewissen und gefährlichen Praeiudicatis kommen, sondern solche zweifelhaftige Fälle an Uns als regierenden König zu Böheimb und deme das ius legis ferendae zustehet, gelangen lassen und darüber Unsern allergnädigsten Aussatz einholen solle. "19 Die Entscheidungsfreiheit des Richters wurde gegenüber D 49 der Landesordnung von 1627 deutlich eingeschränkt, wenn auch vorhandene Gesetze noch nach der ratio ausgelegt werden durften. ${ }^{20}$ Noch unterlagen die richterlichen Anfragen keinem Zwang: Die aus dieser Zeit überlieferten Appellationsgutachten erbitten nämlich vom Kaiser die Entscheidung

\footnotetext{
${ }^{16}$ SPIEGEL, Référé législatif $103 \mathrm{f}$.

${ }^{17}$ Weingarten in seinem Codex FerdinandeoLeopoldino-Josephino-Carolinus, zit. nach SPIEGEL, Référé législatif 105: „allerdings dem buchstäblichen Inhalt der Rechten nachgehen und denen gemäß urteilen sollen".

${ }^{18}$ Sie enthielten als wesentliches Element mehr ständische Freiheit für die Initiative im Landtag, soweit sie die königliche Souveränität nicht beeinträchtigte. 19 Zit. n. SPIEGEL, Référé législatif 105: Ein Appellationsgutachten von 1641 (Weingarten 219) legt dem Kaiser einen "casus dubius et indecisus" zur Entscheidung vor.

${ }^{20}$ Das Reskript von 1661 richtet an die Untergerichte, daß sie „allerdings dem buchstäblichen Inhalt der Rechten nachgehen und denen gemäß urteilen sollen“; SPIEGEL, Référé législatif 105.
} 
von "dubia“, die mit Wohlgefallen beantwortet worden sind: "Gleichwie ihr nun gar recht getan, daß in dergleichen casibus dubiis zu Uns als Legislatorem ihr gehorsamst rekurrieret seiet" (1680). ${ }^{21}$

\section{Codex Theresianus 1758}

a. Ungeachtet der noch zugelassenen Analogie 22 formulierte der Codex Theresianus 1758 in Cap. I §V no. 84 den référé législatif: „Woferne aber dem Richter ein Zweifel vorfiele, ob ein vorkommender Fall in dem Gesetz begriffen seie oder nicht, oder da ihme das Gesetz selbst dunkel schiene, oder ganz besondere und sehr erhebliche Bedenken der Beobachtung des Gesetzes entgegenstünden, so ist die maßgebige Erklärung des Gesetzes allemal bei Uns [d.h. also beim Hof] anzusuchen." $23 \mathrm{Ob}$ diese Anfrage vor oder nach der Entscheidung erfolgen muss, war

\footnotetext{
${ }^{21}$ Vgl. Weingarten 452 (1680): "Gleichwie ihr nun gar recht getan, daß in dergleichen casibus dubiis zu Uns als Legislatorem ihr gehorsamst rekurrieret seiet". (Es handelt sich um die Erklärung des Wortes "Steuern" in Nov. E e $30 \mathrm{im}$ Zusammenhang mit Nov. E e 33.), ferner 560 (1692): „Gleichwie nun daran gar wohl beschehen, daß ihr in diesem zweifelhaften Casu zuvörderst angefraget habet".

22 „Damit Wir jedoch nicht ohne Noth mit Belehrungen über den Verstand Unserer Gesetzen behelliget werden, so gestatten und wollen Wir gnädigst, daß, wann entweder ein bei Gericht anhängiger, in dem Gesetz nicht wörtlich ausgedrückter Fall in allen fürwaltenden Umständen und in der ganzen Beschaffenheit der Sache mit einem in dem Gesetz ausdrücklich entschiedenen Fall vollkommen übereinstimmte [...] oder Unsere höchste Willensmeinung aus der in dem Gesetz klar ausgedrückten Ursache, daß Wir alle nicht buchstäblich berührte Fälle von der nämlichen Beschaffenheit gleichfalls unter dem Gesetz begriffen haben wollen, offenbar erhellet, der Richter sodann ohne fernere Anfrage oder Anstand fürgehen möge und solle." (no. 85 Cap. I § V) HARRASOWSKY, Codex Theresianus 1, 52; siehe auch CONRAD, Richter und Gesetz 35.

${ }^{23}$ HARRASOWSKY, Codex Theresianus 1, 51. Vgl. auch SCHOTT, Rechtsgrundsätze 26f.; CONRAD, Deutsche Rechtsgeschichte 2, 385.
}

nicht geregelt. Diese Regelungen standen im Zusammenhang mit der Wortlautbindung des Richters (no. 81) und der Begrenzung des Billigkeitsrekurses auf gesetzlich vorgesehene Fälle in no. 82 und no. 87 .

b. Die Gesetzgebungsmaterialien belegen die kaiserliche Vorgabe einer richterlichen Vorlagepflicht: Am 10. Dezember 1753 nahm in Brünn eine sog. Compilationskommission ihre Arbeit auf. ${ }^{24}$ Ihr Auftrag war die Bestandsaufnahme des Rechts in den verschiedenen Ländern (Böhmen, Mähren, Schlesien, Niederösterreich, Innerösterreich, Vorderösterreich) ${ }^{25}$ und die Herstellung von Rechtsgleichheit und Rechtssicherheit. Aus der Feder Maria Theresias selbst sollen die Resolutionen für die Kommissionsarbeit vom 3. Mai ${ }^{26}$ und 9. Juni $1753^{27}$ stammen, „dass nebst der Absicht der Gleichförmigkeit deren Gesetzen [...] die in allen Erblanden eingeschlichenen Missbräuche, [...], Schlendrian der soge-

\footnotetext{
${ }^{24}$ HARRASOWSKY, Geschichte der Codification 38f.

${ }^{25}$ Für Böhmen war der Professor und frühere Prager Advocat Joseph Azzoni, für Mähren der Tribunalskanzler Heinrich Hayek von Waldstetten, für Schlesien der Rath von Burmeister, für Niederösterreich der Regierungsrath Joseph Ferdinand Holger und für Innerösterreich der Regierungsrath von Thinnfeld berufen. Für Vorderösterreich kam später Rath von Hormayr hinzu. Zusammensetzung der Kommission in HARRASOWSKY, Geschichte der Codification 40, 44. ${ }^{26}$ Dazu HARRASOWSKY, Geschichte der Codification $48 \mathrm{f}$.
}

${ }^{27}$ Anhand einer am 9.6.1753 durch den obersten Kanzler Graf Haugwitz geleiteten Beratung wird jedoch deutlich, dass durch das Gesetz der Willkür der Justiz gleichzeitig der Kampf angesagt wurde. Den Kompilatoren wurde hier angesichts einer befürchteten ausländischen Kritik geraten, nur im Allgemeinen auszudrücken, dass die Meinung der Kaiserin dahin gehe, ,[...] die schädlichen Kunstgriffe, Hinterlistigkeiten und Schwänke deren Rechtsfreunden abzuschneiden und den gesammten deutschen Erblanden ohne Rücksicht auf alle übrige böhmische und andere Rechte ein gewisses, beständiges, gleiches Recht zu geben, an welches jedermänniglich gebunden sein solle." HARRASOWSKY, Codex Theresianus 1, 28, Anm. 1. 
nannten abusiven Gerichtsordnung $[\ldots]^{\prime \prime 28}$ abgestellt werden sollen. Die Kompilationskommission diskutierte eine richterliche Anfragepflicht nur für landesfürstliche Bestimmungen, während für das gemeine Recht die "autores" benutzt werden sollten. Der Entwurf gesetzlicher Interpretationsregeln durch die Brünner Compilations-Commission ${ }^{29}$ sah zwar keine Analogie mehr vor, beließ aber dem Richter die Freiheit, sich auch am Gesetzessinn zu orientieren. ${ }^{30}$ „Denn unter allen Rechten ist guter Verstand, der von Gott in der Menschen Sinn eingegossen ist, das höchste und fürnehmste Recht.“"31, vernehmen wir Azzoni, den Kommissionsvertreter für Böhmen. Noch in der erneuten Beratung des ersten Hauptstückes am 24. Mai 1757 wurde vor der sogenannten Revisionskommission in Wien, die zur Überarbeitung der Brünner Arbeiten am 9. Juli 1756 eingesetzt worden war, das Argument verhandelt, wonach ein Gesetz nicht alle möglichen Fälle regeln könne. Daher sollte die "interpretatio ex mente seu ratione legis" in den Fällen zugelassen werden, in denen die "ratio in lege klar ausgedrucket" sei. ${ }^{32}$

In der Kritik der Wiener Revisionskommission an den Arbeiten der Brünner Kompilationskommission dominierte die Argumentation der Unvereinbarkeit richterlicher Entscheidungsfreiheit mit Rechtsgleichheit und Rechtssicherheit. Diese entsprang aufgeklärt-absolutistischem Zentralisierungsdenken: Die Verdrängung ständischer Beamte durch landesfürstliche

\footnotetext{
${ }^{28}$ HARRASOWSKY, Geschichte der Codification 48.

${ }^{29} \mathrm{Zu}$ diesem Beratungsvorgang HARRASOWSKY, Codex Theresianus 1, 49f., Anm. 29, 30; DERS., Geschichte der Codification $77 \mathrm{f}$.

${ }^{30}$ Waldstetten zielt ebenfalls in diese Richtung, wenn er betont, dass der Richter nicht auf die bloßen Worte des Gesetzes, sondern auf den eigentlichen Verstand $\mathrm{zu}$ sehen habe. Dabei habe er nur nach der Strenge des Gesetzes und - im Sinne der Gleichheit - nicht nach eigenen Maßregeln zu richten.

${ }^{31}$ HARRASOWSKY, Codex Theresianus 1, 49, Anm. 29.

${ }^{32}$ HARRASOWSKY, Codex Theresianus 1, 51, Anm. 31.
}

und der kontinuierliche Aufbau starker Zentralstellen unter Leitung des Grafen Friedrich Wilhelm von Haugwitz galt auch dem Justizbereich. Mit der Staatsreform von 1749 wurde eine oberste Justizstelle geschaffen, die die alten Hofkanzleien beseitigte und den Ständen im Sektor der Justizverwaltung und Rechtsprechung somit jegliche Mitbestimmung auf der obersten Stufe entzog. ${ }^{33}$ Man brauchte den référé, um eine solche Zentralisierungsbewegung in der Justiz gerade auf der mittleren und unteren Ebene durchzusetzen.

Der von der Revisionskommission 1766 vorgelegte vollständige Entwurf des Codex Theresianus wurde wegen seiner Unübersichtlichkeit und Ungenauigkeit zurückgewiesen. Die Zeitgenossen wollten kein Lehrbuch mehr, das „das gemeine Recht im Gewande der Zeit verarbeitete" ${ }^{\prime 34}$ So forderte die Kaiserin in einem Handschreiben vom 4. August 1772 die Umarbeitung des Gesetzbuches, das durch weitgehende Kürze geprägt und auf grundsätzliche Regelungen beschränkt sein sollte. Es sollte auch nicht zu sehr an das römische Recht gebunden, sondern an der natürlichen Billigkeit orientiert sein..$^{35}$ Die Umarbeitung erfolgte durch den späteren Referenten der Kommission, Hofrat Johann Bernhard Horten. ${ }^{36}$

\footnotetext{
${ }^{33}$ WALTER, Verfassungs- und Verwaltungsgeschichte 101.

${ }^{34}$ CONRAD, Deutsche Rechtsgeschichte 2, 391.

${ }^{35}$ Sтовве, Geschichte der deutschen Rechtsquellen 2, 478.

${ }^{36}$ Neben Horten waren Belser, Zencker, Bourguignon, Holger, Goldegg, Nell und Curti Mitglieder der Kommission. Im Jahr 1773 löste Martini die Mitglieder Goldegg und Froidevo Nell ab. CONRAD, Deutsche Rechtsgeschichte 2, 392; HARROSOWSKY, Codex Theresianus 1, 11f.; DERS., Geschichte der Codification $125 \mathrm{ff}$.
} 


\section{Die Allgemeine Gerichtsordnung von 1781 und das Josephinische Gesetzbuch von 1786}

a. Unter Joseph II., Maria Theresias Sohn und seit 1765 amtierender Kaiser, wurde der erste Teil des revidierten Codex Theresianus durch Patent vom 1. November 1786 als sogenanntes Josephinisches Gesetzbuch publiziert. Schon vorher, am 1. Mai 1781, war die Allgemeine Gerichtsordnung ausgefertigt worden. Beide Kodifikationen - des Zivilprozessrechts 1781 und des Personen- und Familienrechts 1786 sollten nach den Vorgaben der josephinischen (Kompilations-)Kommission ${ }^{37}$ Rechtsvereinheitlichung schaffen, Gleichheit vor dem Gesetz durchsetzen und Standesunterschiede abbauen. Jetzt war es der richterliche Subsumtionsautomat, der für den Erfolg der vernunftrechtlichen Kodifikationen gebraucht wurde. Dementsprechend wurde die richterliche Anfragepflicht in $\S 437$ der Allgemeinen Gerichtsordnung (1781) und in $\S \S 24 \mathrm{ff}$. des ersten Hauptstückes des Josephinischen Gesetzbuches (1786) geregelt.

b. 1781 formulierte $\S 437$ der Allgemeinen Gerichtsordnung folgendes Korsett für die Rechtsanwendung: "1Die Richter sollen verfahren und sprechen nach dem wahren und allgemeinen Verstande der Worte dieses Gesetzes und unter keinem erdenklichen Vorwande eines Unterschiedes zwischen den Worten und dem Sinne des Gesetzes, einer von der Schärfe der Rechte unterschiedenen Billigkeit oder eines widrigen Gebrauchs und dgl. von der klaren Vorschrift dieser Gerichtsordnung abweichen; [...]; ${ }^{3}$ sollte aber über den Verstand des Gesetzes ein gegründeter Zweifel vorfallen, so wird solcher nach Hof anzuzeigen und die Entschließung darüber einzuholen sein; würde aber ein Richter die Streitsachen wider diese Ordnung verzögern oder die Parteien sonst beschweren, so hätte er

\footnotetext{
${ }^{37}$ HARRASOWSKY, Geschichte der Codification $142 \mathrm{ff}$.
} (145); FIJAL, ELLERBROCK, Österreichisches ABGB 521. für allen Schaden zu haften. “38 Für den Richter soll also nur noch das Gesetz maßgebend sein. Billigkeit oder Gewohnheitsrecht sind als Anhaltspunkte diffamiert. Einziger Entscheidungsmaßstab ist der Wortlaut des Gesetzes. Die in den Beratungen zum Codex Theresianus immer wieder präsente Unterscheidung zwischen Worten und Sinn des Gesetzes ist untersagt. Nur die Analogie bei „vollkommene[r] Ähnlichkeit" zwischen einem noch nicht entschiedenen und einem bereits durch das Gesetz erwähnten Fall wird zugelassen: "2nur dann, wenn ein Fall ihm vorkäme, der zwar in dieser Gerichtsordnung nicht entschieden wäre, aber mit einem anderen in selber entschiedenen Falle eine vollkommene Ähnlichkeit hätte, ist dem Richter gestattet, den nicht ausgedrückten Fall nach jener Vorschrift zu entscheiden, die für den ausgedrückten Fall bestimmt ist. [...]“ Adressat der Anfragen war die Hofstelle. Diese kaiserliche Zentralbehörde war als oberste Justizstelle Justizverwaltungsstelle und oberstes Gericht zugleich. Auch die mit den Kodifikationsarbeiten betraute Kompilationskommission sollte für die Beantwortung der Anfragen herangezogen werden können. ${ }^{39} \S 437$ schien sich ausschließlich auf die Bestimmungen der Allgemeinen Gerichtsordnung bezogen $\mathrm{zu}$ haben. Entscheidungen des Hofes wurden nur bei Zweifeln über die Gerichtsordnung, nicht bei sonstigen Anwendungsfragen eingeholt. ${ }^{40}$ Die Justizgesetzsammlung weist eine ganze Reihe von tatsächlich erfolgten Anfragen auf. ${ }^{41}$ Diese Sammlung war angelegt, um durch die Bekanntmachung der Antworten weitere, identische Anfragen $\mathrm{zu}$ vermeiden, und dokumentiert auch von

\footnotetext{
38 Zit. n. SPIEGEL, Référé législatif 106.

39 SPIEGEL, Référé législatif 107.

${ }^{40}$ SPIEGEL, Référé législatif 108, leitet dies anhand von Bestimmungen der Konkursordnung ab.

${ }^{41}$ Nachweise bei SPIEGEL, Référé législatif 108f.
} 
der Hofstelle als offenbar überflüssig angesehene Anfragen.42

c. Durch das Patent vom 1. November 1786 wurde der erste Teil des bürgerlichen Gesetzbuches (Josephinisches Gesetzbuch) kundgemacht. Schon das Patent erneuerte die Anfragepflicht des Richters: "Zugleich werden Richter und Untertanen an den wahren und allgemeinen Verstand der Worte dieses Gesetzes angewiesen und sollen sie unter keinen ersinnlichen Vorwand von der Vorschrift derselben abweichen. Nur wenn dem Richter ein Fall, der in dem Gesetze nicht bestimmt wäre, oder ein gegründeter Zweifel über den Verstand des Gesetzes auffiel, soll die höchste Entschließung durch die vorgesetzte Behörde eingeholt werden. ${ }^{43} \S 26$ des ersten Hauptstückes des Josephinischen Gesetzbuches legte darüber hinaus fest: „Wenn dem Richter ein Zweifel vorfiele, ob ein vorkommender Fall in dem Gesetze begriffen sei oder nicht? Wenn ihm das Gesetz dunkel schiene oder falls besondere und sehr erhebliche Bedenken der Beobachtung desselben entgegenstünden, soll die Belehrung allzeit von dem Landesfürsten gesuchet werden. Aber wenn ein Fall zwar nicht wörtlich in dem Gesetze ausgedrückt, jedoch in den Umständen und der ganzen Beschaffenheit der Sache mit einem anderen ausdrücklich entschiedenen Fall vollkommen gleich wäre, so ist zwar dem Richter den unentschiedenen Fall nach dem entschiedenen zu beurteilen gestattet; doch soll ein solcher Fall jedesmal dem Landesfürsten angezeiget werden." 44 Diese Regelung ist im Zusammenhang mit anderen Bestimmungen zu sehen. Sie ist zusammen mit der Aufhebung aller Gewohnheitsrechte in $\S 9$ und auch $\S 11^{45}$

\footnotetext{
42 SPIEGEL, Référé législatif 108.

${ }^{43}$ Zit. n. SPIEGEL, Référé législatif 109.

${ }^{44}$ Zit. n. SPIEGEL, Référé législatif 110.

${ }^{45}$ Gemäß § 11 ist der Rückgriff auf ein Gewohnheitsrecht nur zulässig, wenn ein Gesetz die Hauptsache entscheidet und es ausschließlich um die Klärung weiterer Umstände geht.
}

oder mit dem Verbot „aller gekünstelten Auslegung der Gesetze, aller Ausdeutung, Erweiterung oder Beschränkung derselben durch Gewohnheiten“ oder "geflissentliche[r] Wortverdrehung" in $\S 25$ zu lesen. Mit dem référé législatif in $\S 26$ legte Joseph II. fest, dass sein Wille und sein Gesetz zur Geltung kommen sollte, und nicht ein unabhängig davon entstandenes Gewohnheitsrecht. ${ }^{46}$ Er sah es als seine Pflicht und als sein Recht an, die Rechte der Untertanen zu bestimmen und dabei jede Quelle der Unsicherheit zu eliminieren.

Schlüsselt man den Wortlaut des $\S 26$ aus dem Josephinischen Gesetzbuch von 1786 auf, wurde der référé législatif in folgenden Fällen vorgeschrieben:

Der Richter stößt auf eine „Lücke“. Hier ist der référé Ersatz der verbotenen Analogie.

Der Richter hält das Gesetz für dunkel. Der référé tritt an die Stelle der dem Richter untersagten und nur dem Monarchen vorbehaltenen Auslegung.

Der Richter hat hinsichtlich der Beobachtung der Gesetze sehr erhebliche bzw. besondere Bedenken. In diesem Fall steht es nur dem Landesherrn zu, Billigkeitserwägungen anzustellen, da er auch das Gnaden- und Dispensionsrecht hat.

Insofern entspricht die Anfrageverpflichtung des $\S 26$ von 1786 den einschlägigen Regelungen des Codex Theresianus (1758) und der Allgemeinen Gerichtsordnung (1781). Ein wesentlicher Unterschied besteht jedoch in der Anzeigepflicht, dass nämlich der Richter nur noch in vollkommen identischen Fällen zur Analogie befugt ist, dies jetzt aber dem Landesfürsten anzeigen muss. Einen weiteren Unterschied bildet die Reichweite der Anfragepflicht. Während der Codex Theresianus und die Allgemeine Gerichtsordnung die Anfragepflicht noch auf das jeweils zugrundeliegende Gesetz beziehen,

46 SPIEGEL, Référé législatif 111. 
spricht das erste Hauptstück des Josephinischen Gesetzbuches dagegen „von den Gesetzen“47 und erweitert so die richterliche Vorlagepflicht auf alle Rechtsnormen. In diesem Zusammenhang stellt $\S 10$ des Josephinischen Gesetzbuches klar: "Auch in denjenigen Fällen, worüber in den Gesetzen nichts verordnet ist, ist keine Gewohnheit zulässig [...] Soll in dergleichen Fällen eine allgemeine und gewisse Anordnung für notwendig $[\ldots]$ gehalten werden, ist dieselbe bei dem Landesfürsten zu suchen. “48

Den mit dem Josephinischen Gesetzbuch erreichten Höhepunkt judizieller Restriktion ${ }^{49}$ belegt eindrucksvoll ein Hofdekret vom 7. März 1787. Die Anfrage des böhmischen Appellationsgerichts, ob Cap. I $\S 26$ des ersten Teils des Josephinischen Gesetzbuches den § 437 der Allgemeinen Gerichtsordnung verdrängt habe, ${ }^{50}$ dokumentiert zunächst die verängstigte Beachtung der Anfragepflicht durch die Gerichte. Die Antwort der Hofstelle ließ den Gerichten die Freiheit, dass hinsichtlich einer möglichen Analogie bei zwei identischen Rechtsfällen „der Richter allerdings mit Schöpfung und Kundmachung des Urteils vorgehen" könne. Dies erlaubt den Umkehrschluss, dass viele Richter sich scheuten, selber zu beurteilen, ob ein solcher zur Analogie berechtigender - Fall auch tatsächlich vorliege..$^{51}$

d. Die Beratungen zum Josephinischen Gesetzbuch dokumentieren ein Ringen in der Kompilationskommission um eine richterliche Restfreiheit in Form von Interpretationsregeln: Aus der Sitzung der Compilations-Kommission vom 13. Oktober 1772 ist der Protest von Goldegg

\footnotetext{
${ }^{47}$ SPIEGEL, Référé législatif 109.

48 Zit. n. SPIEGEL, Référé législatif 110.

${ }^{49}$ So auch SCHOTT, Rechtsgrundsätze 29.

50 Zit. n. SPIEGEL, Référé législatif 112.

${ }^{51}$ Es gab auch Anfragen, die sich mit der Auslegung des 1787 eingeführten Strafgesetzes befassten, welches die Anfragebestimmung nicht ausdrücklich enthält, da der erste Teil dies bereits ausdrückt. Dazu SPIEGEL, Référé législatif $112 \mathrm{f}$.
}

gegen die ihm zu weitgehende Beschränkung der richterlichen Auslegung überliefert. ${ }^{52}$ Sein Gegenvorschlag gestattete den Richtern, dasjenige Gesetz anzuwenden, „welches nach der natürlichen Billigkeit oder nach dem Sinn Unserer Gesetze sich am besten zur Entscheidung schicket". Gleichzeitig sollten die Analogiebestimmungen, die sich auf "gleiche" Fälle bezogen, auf „ähnliche“ erweitert werden. Goldegg begründete seinen Vorschlag mit der Feststellung, dass es trotz der vielen Detailbestimmungen nur wenige Fälle gebe, die ausschließlich aus dem Wortlaut des Gesetzes gelöst werden könnten. So sei man häufig genötigt, die Entscheidung „entweder aus der Ursach oder Sinn des Gesetzes oder Combination der Gesetzen selbst oder aus denen allgemeinen Grundregeln“ zu entnehmen. Da das zukünftige Gesetz sich nach den aufgestellten Grundsätzen nicht im Detail verlieren solle, seien Interpretationsregeln umso wichtiger." Durch die Anfragepflicht befürchtete Goldegg eine die Parteien kostenmäßig benachteiligende Verlagerung in die oberste Instanz. Die Vielzahl von Anfragen würde den Landesfürsten unnötig behelligen und $\mathrm{zu}$ obrigkeitlichen Gesetzeserläuterungen führen, die die Digesten überträfen.

Die Gesetzeskommission sprach sich jedoch gegen die Aufstellung entsprechender Interpretationsregeln auf, da "[...] dem Richter unmöglich eine interpretatio legis eingeraumet werden könne, ohne die ganze bei Verfassung des neuen Gesetzbuches gehegte Absicht zu vereiteln." ${ }^{\text {"53 }}$ Dies billigte am 18. November 1772 auch der Kaiser mit der "gehegte[n] Absicht", wonach das Gesetz den Willen des Monarchen ausdrücke und der Richter diesen durch strikte Rechtsanwendung $\mathrm{zu}$ verwirklichen habe, ohne ihn durch eigene Interpretation verfälschen zu können. Und als Hortens Entwurf für die Fälle, in

\footnotetext{
${ }^{52}$ Harrasowsky, Codex Theresianus 4, 22, Anm. 17. Dort sind die nachfolgenden Zitate belegt.

${ }^{53}$ HarrasowsKy, Codex Theresianus 4, 22, Anm. 17.
} 
denen ein Fall aus einem vollkommen gleichen Fall entschieden werden konnte, noch keine Anzeigeverpflichtung vorsah, statuierte man auch diese, um „willkürlichen Auslegungen der Gesetze zu begegnen. ${ }^{454}$ Horten hatte sich zuvor gegen diese Anordnung ausgesprochen, weil er eine übermäßige Zahl an Anzeigen befürchtete.

Fasst man die Regelungen der Allgemeinen Gerichtsordnung 1781 und des Josephinischen Gesetzbuches 1786 zusammen, erscheint der référé législatif als Garant des obrigkeitsstaatlichen Gesetzgeberwillens und zum Vehikel für Rechtssicherheit und Rechtseinheit. Das mitschwingende tiefe Misstrauen gegenüber Juristen ${ }^{55}$ passt zur Verdrängung der ständischen Richter. So setzte Joseph II. die Zentralisierung, die bereits unter Maria Theresia auf der obersten Stufe des Gerichtswesens stattgefunden hatte, innerhalb der unteren Instanzen fort, ${ }^{56}$ in denen es aufgrund der territorialen Entwicklung und der ständischen Aufspaltung starke Unterschiede gab. Durch die Allgemeine Gerichtsordnung von 1781 führte er einen allgemeinen dreizügigen Instanzenzug ein, errichtete Appellationsgerichte in den einzelnen Ländern und beseitigte die Verschiedenheit des Gerichtsstandes für Adlige und Nichtadlige. ${ }^{57}$ Um sich der Gefolgschaft der Beamten und Untertanen sicher sein zu können, schuf er sich zudem einen mächtigen Polizeiapparat. ${ }^{58}$ Aus diesem Kontext erklärt sich die schärfste Ausprägung des référé innerhalb der österreichischen Rechtsgeschichte. Nur eine Ausgestaltung der richterlichen Vorlagepflicht wie im Normtext des $\S 26$ ermöglichte

\footnotetext{
${ }^{54}$ HARRASOWSKY, Codex Theresianus 4, 22, Anm. 17.

${ }^{55}$ HARRASOWSKY, Geschichte der Codification 41.

56 WALTER, Österreichische Verfassungs- und Verwaltungsgeschichte 109f.; HATTENHAUER, Geschichte $180 \mathrm{ff}$.

57 WALTER, Österreichische Verfassungs- und Verwaltungsgeschichte 111. Ausgenommen war die unterste Stufe.

${ }^{58}$ WALTER, Österreichische Verfassungs- und Verwaltungsgeschichte 112; HATTENHAUER, Geschichte 185f.
}

die Zentralisierung der Rechtsprechung und die Rekrutierung folgsamer Richter.

\section{Das Patent von 1791}

Mit dem Patent vom 22. Februar 1791 wurde der référé législatif i.d.F. des Josephinischen Gesetzbuches von 1786 geändert. ${ }^{59}$ Dieses sog. Februarpatent enthielt das Eingeständnis, dass die wohlgemeinten Absichten des Gesetzbuches aus dem Jahre 1786 nicht erreicht worden sind und Gegenstand vielfältiger Klagen seit dem Antritt Leopolds II. waren, so dass man sich zu der "dringendsten Abänderung bewogen gefunden“ habe. Diese wurden in acht Paragraphen umgesetzt. $\S 2$ des Februarpatentes „Von dem richterlichen Urteile nach den Gesetzen" bestimmte folgendes: „Anstatt des 26. Absatzes des ersten Hauptstückes, welcher hiermit aufgehoben wird, wird verordnet: daß der Richter, wenn er einen vorkommenden Fall nicht in den Worten des Gesetzes entschieden fände, auf den zusammenstimmenden Begriff und Sinn desselben, auf gleichförmige darin ausgedrückte Fälle, auf die aus der Verbindung der Gesetze sich darstellenden Grundsätze und Absichten sehen und den Fall nach derselben Maßgebung beurteilen soll. Stünden der Beobachtung des Gesetzes besondere und sehr erhebliche Bedenken entgegen, so wäre die Belehrung bei Hof anzusuchen." ${ }^{60}$ Dem Richter wurden die Auslegung und analoge Anwendung der Gesetzesbestimmungen gestattet. Von den drei Anfragekomplexen des Josephinischen Gesetzbuches blieb nur noch der Fall des Abweichens vom Gesetz unter Billigkeitserwägungen, der auch weiterhin dem Landesherrn vorbehalten bleiben sollte. Da Lücken und Zweifel des Richters bei Anwendung des Gesetzes keine Gründe mehr für einen

\footnotetext{
${ }^{59}$ Eine von Kaiser Leopold II. 1790 eingesetzte Kommission befasste sich mit der Umarbeitung des Josephinischen Gesetzbuches, HARRASOWSKY, Geschichte der Codification 152f.

60 Zit. n. SpIEGEL, Référé législatif 114.
} 
référé législatif darstellten, erfuhr das Institut durch das Patent eine starke Einschränkung, wenn nicht sogar seine gesetzliche Aufhebung.

Zur Interpretation des Patentes vom 22. Februar 1791 berichten die Quellen folgendes: Bereits am 4. Januar 1787 hatte es in einer Sitzung der obersten Justizstelle über die Behandlung von Anfragen Meinungsverschiedenheiten gegeben. ${ }^{61}$ Uneinigkeit bestand insbesondere darüber, ob alle Anfragen an die Kompilationskommission weitergeleitet werden sollten oder nur solche, die sich nach Befinden der Justizstelle nicht ausschließlich aus den Worten des Gesetzes entnehmen ließen. ${ }^{62}$ Zudem war strittig, ob die oberste Justizstelle die weitergeleiteten Anfragen mit einem Gutachten versehen sollte, da der böhmische und polnische Senat der obersten Justizstelle immer wieder vorgeworfen hatte, die Rechtsgrundlagen nicht genau zu kennen. ${ }^{63}$ Der (materiellrechtlich fundierte) Umgang mit den Anfragen wurde auch in der Sitzung der neuen - unter dem Präsidium des Wiener Naturrechtslehrers Karl Anton von Martini zu Wasserberg - einberufenen Kommission am 5. Juni 1790 durch den Referenten Lewinski problematisiert. Lewinski wies auf die Verwirrungen hin, die durch den „Wust von Erläuterungen" entstehen mussten. In den Protokollauszügen ist folgende Aussage von ihm bezüglich der Anfragepflicht überliefert: „Durch diesen $\S$ habe man alle Jurisprudenz verbannt, er mache die Richter alle zu Maschinen und öffne der Ignoranz Thür und Angel, weil sie sich nur an den Buchstaben anhalten darf, womit schon Alles gut ist." ${ }^{\prime 64}$ Dieser zaghaften Rückbesinnung auf die richterliche Entscheidungsfreiheit zum

\footnotetext{
${ }^{61}$ Harrasowsky, Codex Theresianus 4, 22, Anm. 17.

62 Unabhängig davon lässt sich hier schon die Entwicklung erkennen, wonach die Kompilationskommission allmählich ihre Aufgaben auf alle Zweige der Justizgesetzgebung erweiterte.

${ }^{63}$ Letztere sprachen sich deshalb für die Weiterleitung aller Anfragen ohne Gutachten aus.

${ }^{64}$ HARRASOWSKY, Codex Theresianus 4, 22, Anm. 17.
}

Trotz belegen Einträge in die Justizgesetzsammlung auch noch nach 1791, dass es auch nach dem Februarpatent Anfragen gab, die sich weiter auf den Inhalt der älteren Bestimmungen stützten. ${ }^{5}$ Das definitive Ende des référé législatif im österreichischen Recht leiteten erst die Beratungen des Urentwurfes des Westgalizischen Gesetzbuches in der Hofkommission 1792-1794 ein.

\section{Das Westgalizische Gesetzbuch 1798}

Die Hofkommission vollendete unter Franz I. in den Jahren 1794-1796 den Entwurf eines neuen Gesetzbuches, welches als sogenanntes Westgalizisches Gesetzbuch (WGGB) im Jahr 1798 in West- und Ostgalizien eingeführt wurde. Ein Entwurf v. Martinis zu $\S \S 18,19$ WGGB schwächte die Anfragepflicht aus dem Februarpatent 1791 so weit ab, dass die Vorlagepflicht zweifelhafter Rechtsfälle vollkommen beseitigt wurde. Seine Vorlage des $\S 18$ WGGB bestimmte, dass der Richter bei der Auslegung und Anwendung des Gesetzes diesem keinen anderen Sinn beilegen dürfe als den, der nach den Regeln der Sprache aus der Bedeutung der Worte und ihres Zusammenhanges oder aus der klaren Absicht des Gesetzgebers deutlich hervorleuchte. V. Martinis Vorschlag für WGGB I § 19 formulierte: „Findet aber der Richter einen Rechtsfall durch die Worte des Gesetzes nicht geradezu entschieden, so muß er in seinem Urtheile auf den natürlichen Sinn des Gesetzes, er muß ferner auf die Gründe anderer damit verwandten Gesetze, und auf ähnliche im Gesetze bestimmt entschiedene Fälle Rücksicht nehmen; bleibt ihm der Rechtsfall nach allem diesem noch zweifelhaft, so muß er ihn mit Hinsicht auf die sorgfältig gesammelten, und reiflich erwogenen Sachumstände nach den allgemeinen und natürli-

\footnotetext{
${ }^{65}$ Dazu Wellspacher, Naturrecht und ABGB 185; SPIEGEL, Référé législatif 114, der durch das Patent die eigentliche Aufhebung des référé législatif in Österreich sieht.
} 
chen Rechtsgrundsätzen entscheiden.“66 Der Richter wurde also mit den Regelungen von 1798 bei Zweifeln auf die "allgemeinen und natürlichen Rechtsgrundsätze“ verwiesen.

Die Beratungen von 1792 bis 1794, die in den Jahren 1796/97 in die §§18, 19 des WGGB mündeten, zeigten noch einmal die vielfältigen Argumente der Befürworter und Gegner des référé législatif. ${ }^{67}$. In ihren Stellungnahmen zu dem 1792 vorgelegten Entwurf wiesen besonders die Stände von Tirol, Schlesien, Krain und die oberösterreichischen Stände sowie der Magistrat in Innsbruck und die Kommission in Kärnten auf die Gefahr hin, die sich aus einer dem Sinn des Gesetzes folgenden Auslegung ergeben könnte. ${ }^{68}$ Dem Richter würde so eine zu große Freiheit gewährt, und „[es würde] eine neue Quelle für die Ränke der Advokaten eröffnet". So sprach man sich dagegen aus, dass frühere Urteile zur Auslegung eines Gesetzes herangezogen werden können, um das Entstehen von Gewohnheiten aus gleichförmigen Rechtssprüchen auszuschließen. Stattdessen wurde eine Anzeige- oder Anfragepflicht vorgeschlagen.

Die Stände von Kärnten und Niederösterreich machten dagegen zusammen mit dem Prager Professor Groß Front gegen eine derartige Einschränkung der richterlichen Tätigkeit. Der Vertreter der Kärntener Stände verwies auf die Fälle, „wo eine allzu genaue Beobachtung des Buchstabens des Gesetzes darum nicht möglich sei, weil dadurch der Endzweck des Gesetzes verfehlt werde, zu dessen Erreichung doch eigentlich das Gesetz gegeben worden sei." ${ }^{69}$ Die Stände von Niederösterreich wollten dem Richter gestatten, „bei einem sich äußernden Unterschiede zwischen den Worten des Gesetzes und

\footnotetext{
${ }^{66}$ HARRASOWSKY, Geschichte der Codification $155 \mathrm{f}$.

${ }^{67}$ HARRASOWSKY, Codex Theresianus 4, 10f.; 5, 10f., Anm. 6; DERS., Geschichte der Codification 152ff.; OFNER, Ur-Entwurf 1, 3f.

${ }^{68}$ HARRASOWSKY, Codex Theresianus 5, 10, Anm. 6.

${ }^{69}$ Harrasowsky, Codex Theresianus 5, 10, Anm. 6.
}

dem auffallenden Sinne desselben sich nach diesem letzteren $\mathrm{zu}$ benehmen, auf gleiche Art von der wörtlichen gesetzlichen Vorschrift und der hierin enthaltenen Strenge der Rechte abzuweichen, und vielmehr der natürlichen Billigkeit Platz zu geben, wenn diese Billigkeit mit dem Sinne jener Vorschrift ungezweifelt vereinbarlich ist. “70 Die Bindung an den Wortlaut des Gesetzes könnte einen Widerspruch zu dem Gesetzgeberwillen erzeugen. Ferner würden die Richter zu einem "mechanischen Werkzeug" und die "tüchtigen“, „,aufgeklärten“ und "unparteiischen" Richter müssten sich auf diese Weise gelähmt fühlen. Der Prager Professor Groß wies noch einmal darauf hin, dass es unmöglich sei, alle Fälle im Gesetz zu entscheiden.

Hofrat Keeß, der maßgeblichen Anteil an den Bestimmungen des Josephinischen Gesetzbuches gehabt hatte ${ }^{71}$ und nun die Begutachtungen des Entwurfs eines Westgalizischen Gesetzbuches verarbeiten sollte, setzte sich dafür ein, dass sich die Antworten auf die gestellten Anfragen nur auf zukünftige Fälle beziehen und nie anhängige Rechtssachen aufhalten sollten. ${ }^{72} \mathrm{Er}$ kritisierte die Vorschläge, die darauf abzielten, unter Hinweis auf den Sinn des Gesetzes vom Wortlaut desselben abweichen zu können, weil hierdurch die Gleichförmigkeit der Gesetzesanwendung erheblich gefährdet sei. ${ }^{73}$ Die richterliche Entscheidung dürfe zudem nicht an der Billigkeit ausgerichtet werden, diese diene lediglich dem Landesherrn als Maßstab bei der Gesetzgebung. Sei das Gesetz einmal gegeben, dürfe niemand mehr das Recht haben, sich

\footnotetext{
${ }^{70}$ HARrASOWSKY, Codex Theresianus, 5, 10 Anm. 6. ${ }^{71} \mathrm{Zu}$ den damit entstehenden Veränderungen und Verbindungen HARRASOWSKY, Codex Theresianus 4, 9.

72 Harrasowsky, Codex Theresianus 5, 11, Anm. 6.

${ }^{73} \mathrm{Zu}$ Keeß Bezüge auf England vgl. PETER, Englisches Recht 928.
} 
durch die Berufung auf die Billigkeit der Anordnung des Gesetzgebers zu entziehen. ${ }^{74}$

Hinsichtlich künftiger Anfragen erklärte Hofrat Keeß: „Es gehört nur Gelassenheit und Geduld dazu, sie zu belehren; die Vollkommenheit ist immer nur ein Werk der Zeit."75 Diese Worte scheinen noch in der Tradition des $\S 26$ des Josephinischen Gesetzbuches von 1786 zu stehen. Dennoch geht Keeß zu den Regelungen dieses Gesetzbuches auf Distanz, wenn er die Antworten auf richterliche Anfragen nur noch auf zukünftige Fälle anwenden möchte. Seine Motive lassen sich in den Quellen nicht genau klären. Es ist aber zu vermuten, dass sie mit der Prozessverzögerung in Verbindung stehen. ${ }^{76}$

In der Beratung vom 10. August wurde der Entwurf v. Martinis dahingehend ergänzt, dass der Richter in einem Fall, der durch die Worte des Gesetzes nicht entschieden ist, auf „den erklärten Sinn desselben" verwiesen wurde, da man die „von dem Richter selbst gegebenen rationes legis nicht gelten" lassen könne. Die Erklärung eines Gesetzes könne nur dem Gesetzgeber überlassen bleiben. Zudem erkannte man ausschließlich die „allgemeinen Rechtsgrundsätze“, also das allgemeine und Provinzialrecht als Subsidiärquelle an und verwies nicht auf das von Martini angeführte Naturrecht, „weil die Lehrer des Naturrechts in gewissen Grundsätzen unter sich selbst nicht einig sind“. Ergebnis dieser Beratungen sind dann die $\S \S 18,19$ WGGB. ${ }^{77}$ Daher ist m.E. der von Lukas aufge-

\footnotetext{
${ }^{74}$ Unter Berufung auf Montesquieu verwies er darauf, dass einige Dinge, welche für den Einzelnen als unbillig erscheinen, im Interesse der Gesamtheit notwendig seien. (HARRASOWSKY, Codex Theresianus 5, 11, Anm. 6.)

${ }^{75}$ HARRASOWSKY, Codex Theresianus 5, 11, Anm. 6.

${ }^{76}$ HarrasowsKy, Codex Theresianus 5, 11, Anm. 6: Dort wird betont, dass Keeß sich für die Anwendung auf zukünftige Fälle aussprach, da die Antworten „nie die Entscheidung eines anhängigen Falles aufhalten dürfen".

${ }_{77}$ MiERSCH, Sogenannter référé législatif 48.
}

stellten These zu widersprechen, wonach der Verweis auf das Naturrecht in Österreich die Möglichkeit der Aufhebung des référé législatif eröffnet.

\section{Die Beratungen vom Urentwurf des WGGB zum ABGB}

Nach Abschluss der Beratungen beschloss die Kommission, dem Kaiser das Ergebnis des ersten Teils mit der Bitte um Erteilung der Sanktion vorzulegen. In einem Handschreiben vom 21. Juli 1796 ordnete der Kaiser jedoch erneut eine „Directions-Commission" an, die aus höheren Verwaltungsbeamten bestehen und den vorgelegten Gesetzentwurf überprüfen sollte, so dass faktisch eine neue Revisions-Kommission eingerichtet wurde ${ }^{78}$. In der Sitzung vom 11. Januar sprach sich die "DirectionsCommission" gegen die Möglichkeit des Richters aus, im Zweifel über die Anwendung eines Gesetzes nach allgemeinen Rechtsgrundsätzen entscheiden $\mathrm{zu}$ können. ${ }^{79}$ Ferner wandte man sich gegen die Möglichkeit, die Auslegung auf die Absicht des Gesetzgebers zu stützen. Man äußerte die Sorge, dass damit der richterlichen Willkür wieder ein weiter Spielraum eröffnet werde. So wurde mehrheitlich beschlossen, den Richter im Zweifel bei dem Gesetzgeber anfragen zu lassen. Um nicht von Anfragen zu sehr überhäuft zu werden, sollten die Obergerichte ermächtigt werden, ungerechtfertigte Anfragen unter Anführung der einschlägigen Gesetzesstellen zurückzuweisen.

Gegen diese Ansicht stellte sich das Kommissionsmitglied Fechtig, der erklärte, dass dadurch die Obergerichte genötigt würden, ihre im Instanzenzug abzugebenden Entscheidungen zu präjudizieren. Zudem befürchtete er Verzögerung der Rechtspflege. Er sprach auch von der Unmöglichkeit, für alle Fälle ausreichende und

\footnotetext{
${ }^{78}$ HARRASOWSKY, Codex Theresianus 4, 11.

${ }^{79}$ Harrasowsky, Codex Theresianus 5, 11, Anm. 6.
} 
erschöpfende Gesetze zu geben. Der Richter müsse vielmehr an ein Subsidiarrecht verwiesen werden. Seine positiv-rechtliche Haltung kam dadurch zum Ausdruck, dass er als Subsidiarrecht nicht das Naturrecht, sondern das bestehende allgemeine und Provinzialrecht nannte, da einzig dieses dem Volke bekannt sei und dort nicht außer Kraft gesetzt werden könne, wo das neue Gesetz Lücken aufweise. Um den Gesetzgeber auf die Lücken aufmerksam zu machen, schlug er eine Anzeigepflicht in allen Fällen vor, die nicht aufgrund des bürgerlichen Gesetzbuches entschieden werden konnten. In Abständen von fünf Jahren könne dann die Gesetzgebung die erforderlichen Regelungen treffen.

Die Stellungnahmen der Revisions-Commission führten nicht direkt zu neuen Gesetzentwürfen. Ende des Jahres 1796 hatte die Gesetzgebungskommission den zweiten und dritten Teil des gesamten Gesetzeswerks fertiggestellt, während die Revisions-Commission über das dritte Stück des ersten Teils nicht hinausgekommen war. Die Gesetzgebungskommission drang darauf, den kompletten Entwurf in den einzelnen Ländern begutachten zu lassen. Ehe der Entwurf an die Länderkommissionen abgeschickt wurde, führte man diesen in Westgalizien am 13. Februar 1797 als Gesetz ein. Im Jahre 1797 wurde der Entwurf 17 Kommissionen zur Beurteilung vorgelegt. ${ }^{80}$

Erst im Jahre 1801, nachdem alle Gutachten eingegangen waren, konnte die neu eingesetzte Kommission ihre Tätigkeit beginnen, in der der Appellationsrat und Professor des Naturrechts und des römischen Rechts Franz Anton von

\footnotetext{
80 OFNER, Ur-Entwurf 11: Nach dem Vortrag des Referenten v. Zeillers bildeten diese 17 Kommissionen: das niederösterreichische, innerösterreichische, oberösterreichische, böhmische, mährisch-schlesische und ostgalizische Appellationsgericht, dann die Landrechte in Österreich ob der Enns, Steiermark, Krain, Görz, Triest, zu Stanislau und Tarnow und die Fakultäten in Wien, Prag, Innsbruck und Freiburg. Hierzu auch und besonders zum Votum der Freiburger Universität: SCHOTT Rechtsgrundsätze $16 \mathrm{ff}$.
}

Zeiller das Referat führte. Den Vorsitz übernahm zunächst der Staatsminister Graf von Rottenhann und nach dessen Tode der Oberstlandrichter von Haan. In der ersten Sitzung am 21. Dezember 1801 referierte Zeiller in einem Vortrag die Anforderungen an das neue Gesetzeswerk. ${ }^{81}$ Die erste und eigentliche Bestimmung der Kodifikation sei es, den Bürger über Recht und Unrecht im Voraus zu belehren. In der augenblicklichen Situation sei dies nicht möglich, da selbst dem gebildeten Bürger in den meisten rechtlichen Geschäften nichts anderes übrig bleibe, als sich der „unsicheren und eigenmächtigen Leitung eines Rechtsfreundes" blindlings anzuvertrauen. Die gelehrten Meinungsstreitigkeiten zur römischen Gesetzessammlung machten es dem "Rechtsfreunde“ leicht, jedes noch so bedächtlich geschlossene Geschäft in einen Rechtszweifel zu ziehen. ${ }^{82}$ Deshalb müssten die Gesetze vollständig sein, damit sich kein Rechtsfall ereignen könnte, der nicht aus dem Gesetz gelöst werden könne. Zeiller räumte jedoch ein, dass jede Gesetzgebung scheitern müsse, wenn sie jede in dem Geist des Gesetzes und in allgemeinen Rechtsprinzipien gegründete Auslegung verbiete, die Richter zu Maschinen mache und diese nur noch an den Wortlaut der Gesetze verweise. Wie jedoch die Willkür des Richters bei dem Verweis auf allgemeine Rechtsgrundsätze vermieden und die eigenmächtige Auslegung verhindert werden könnten, müsse die Kommission bei den Beratungen über die Auslegung der Gesetze regeln. ${ }^{83}$ Die erste Beratung über die Auslegungsregeln fand am 4. Januar 1802 statt. $^{84}$ Zunächst wurde auf die vorangehend dargestellte Beratung in der Revisions-Commission verwiesen. Zeiller konzentrierte seine Argumentation darauf, zur Vermeidung richterlicher Willkür diesen nicht

\footnotetext{
${ }^{81}$ OFNER, Ur-Entwurf 1, 1ff.

82 OFNER, Ur-Entwurf 1, 4f.

83 OFNER, Ur-Entwurf 1, 6.

${ }^{84}$ OFNER, Ur-Entwurf 1, 22.
} 
nach der Absicht des Gesetzgebers oder nach den natürlichen Rechtsgrundsätzen entscheiden zu lassen. Damit ging er auf einen Vorschlag der Freiburger Fakultät nicht ein, ${ }^{85}$ die einen Rückgriff auf die allgemeinen und natürlichen Rechtsgrundsätze durch den Richter auch in den Fällen zuließ, ,[...] wenn aus der wörtlichen Auslegung eines an sich zwar klaren Gesetzes wegen vorkommender, ganz besonderer, vom Gesetzgeber nicht vorhergesehenen Umstände eine offenbare Ungerechtigkeit oder Ungereimtheit folgen sollte." 86 Für Zeiller war der Rückgriff auf die allgemeinen und natürlichen Rechtsgrundsätze ein Übel, da der Richter kein gründlicher Rechtsphilosoph sei. ${ }^{87}$

In der Darstellung der Beratungsprotokolle bei Ofner finden sich drei Diskussionslinien im Spannungsfeld zwischen richterlicher Entscheidungsfreiheit und Missbrauchskontrolle:

(1.) Der Vorschlag, nicht ausdrücklich geregelte Fälle nach den Grundsätzen des gemeinen oder des Provinzialrechts $\mathrm{zu}$ entscheiden, wird mit den Argumenten verworfen, dass das gemeine Recht in Form des römischen Rechts durch das neue Gesetzbuch gerade aufgehoben werden sollte und dass ein Provinzialrecht ohnehin den Vorzug vor dem natürlichen Recht habe. (2.) Der Gedanke einer Vorlagepflicht bei der nächsten Instanz wird mit den Argumenten der ohnehin gegebenen Appellationsmöglichkeit verworfen: die Parteien verlören sonst eine zweite Instanz, der zweite Richter wäre nicht mehr unbefangen und der erstinstanzliche Richter müsse möglicherweise gegen seine Überzeugung eine Entscheidung treffen, wenn er vor seiner eigenen Entscheidung die eines Oberrich-

\footnotetext{
${ }^{85}$ Siehe SCHOTT, Rechtsgrundsätze 18, der auch zu dieser Feststellung gelangt. Einzelheiten über die Mitglieder der Freiburger Kommission, DERS., $20 \mathrm{ff}$.

${ }^{86}$ SCHOTT, Rechtsgrundsätze 18.

${ }^{87}$ Zeiller geht es um die Begrenzung möglichen Missbrauches (OFNER, Ur-Entwurf 1, 23; SCHOTT, Rechtsgrundsätze 30).
}

ters einzuholen hätte.

(3) Auch die Vorlage an die oberste Gerichtsbehörde oder an den Gesetzgeber wird verworfen: „Die nämlichen Berathungen streiten wider den weiteren Vorschlag, die Akten von Amtswegen an die oberste Justizbehörde zu geben; und sie streiten in noch größerem Maße wider den Vorschlag, die Entscheidung des Gesetzgebers oder der Gesetzkommission einzuholen. Die Parteien hätten dann nur mehr eine Instanz und der gesetzgebende Körper würde zugleich der richtende sein." 88 Auch Zeillers Vorstoß, eine Anzeigepflicht für die Fälle anzuordnen, „die aus den natürlichen Rechtsgrundsätzen entschieden werden und keinem weiteren Rechtszuge unterliegen", 89 setzte sich im Ergebnis nicht durch. Weder das Kundmachungspatent vom 1. Juni 1811 noch die gesetzlichen Bestimmungen der $\S \S 7,8$ ABGB erhielten eine Anzeigeverpflichtung. Im Ringen um Rechtssicherheit, Rechtseinheit, Verhütung von Willkür und Missbrauch, um Interpretationsregeln und Regelungslücken setzte sich in den Beratungen die Einsicht durch, dass der référé législatif zu einem nicht hinnehmbaren Zustand führe: Wie es bedenklich sei, den Richtern einen zu großen Spielraum zu belassen, so sei es demgegenüber für die Rechtspflege sogar gefährlich, wenn man die Richter, wie im Josephinischen Gesetzbuche, auf den bloßen Buchstaben verpflichte. ${ }^{90}$ Neben der Prozessverzögerung ${ }^{91}$ und der Instanzen-

\footnotetext{
88 OFNER, Ur-Entwurf 1, 23.

${ }^{89}$ Zeillers Vorschlag berücksichtigte auch noch weitere redaktionelle Änderungen. So sollte u.a. in $§ 19$ nach Anregung der Universität Innsbruck auf den Begriff „allgemeine" Rechtsgrundsätze verzichtet werden, weil diese leicht als Verweis auf die römischen Regeln gewertet werden könnten - dazu OFNER, Ur-Entwurf 1, 23.

90 OfNER, Ur-Entwurf 1, 24, der weitere Punkte der übrigen Kommissionsmitglieder benennt.

${ }^{91}$ SPIEGEL, Référé législatif 115 führt die Entlastung der Justizstelle auch als Grund an, wendet sich aber gegen LUKAS, Zur Lehre 422, 425, wonach die Beschleunigung der Rechtspflege Grund war, da die
} 
verkürzung waren es die Notwendigkeit richterlicher Entscheidungsfreiheit und die Unvereinbarkeit von richterlicher und gesetzgebender Funktion, die zur Aufhebung des référé législatif führte. ${ }^{22}$ Das natürliche Recht war dabei laut Zeillers Kommentar von 1811 ein „unversiegbarer Quell“, so dass die Vollständigkeit der Rechtsordnung immer gewährleistet war. ${ }^{93}$

Nachdem das Gesetzbuch an die inländischen Juristenfakultäten und an eigens geschaffene Kommissionen in den deutschen Provinzen zur Begutachtung verschickt worden war, wurde ein mehrmals revidierter Entwurf am 1. Juni 1811 als Allgemeines Bürgerliches Gesetzbuch für die gesamten deutschen Erbländer publiziert. ${ }^{94}$ Der noch heute gültige $\S 7$ ABGB erhielt folgende Fassung: „Läßt sich ein Rechtsfall weder aus den Worten, noch aus dem natürlichen Sinne eines Gesetzes entscheiden, so muß auf ähnliche, in den Gesetzen bestimmt entschiedene Fälle, und auf die Gründe anderer damit verwandten Gesetze Rücksicht genommen werden. Bleibt der Rechtsfall noch zweifelhaft; so muß solcher mit Hinsicht auf die sorgfältig gesammelten und reiflich erwogenen Umstände nach den natürlichen Rechtsgrundsätzen entschieden werden. ${ }^{495}$ Die authentische Interpretation behielt $\S 8$ ABGB dem Gesetzgeber vor: „Nur dem Gesetzgeber steht die Macht zu, ein Gesetz auf eine allgemein verbindliche Art zu erklären. Eine solche Erklärung muß auf alle noch zu entscheidende Rechtsfälle angewendet werden, dafern der Gesetzgeber nicht hinzufügt, dass seine Erklärung bei Entscheidung solcher Rechtsfälle, welche die vor der Erklärung unternommenen Handlungen und angesprochenen

Hofstelle Anfragen „ziemlich rasch erledigte" (m.w.N.).

${ }^{2}$ Dazu auch SpIEgel, Référé législatif 116, der von "geläuterten wissenschaftlichen Anschauungen" Martinis und Zeillers spricht.

${ }_{93}$ ZEILLER, Commentar 21, 66.

${ }^{94}$ Zit. n. OFNER, Ur-Entwurf 1, 4.

95 Dazu OfNer, Ur-Entwurf 1, $1 \mathrm{ff}$.
Rechte zum Gegenstande haben, nicht bezogen werden solle. ${ }^{\prime 96}$

\section{Zusammenfassung der öster- reichischen Entstehungsgeschichte des référé législatif}

Der référé législatif kommt in Österreich vom 17. bis in das 19. Jahrhundert in unterschiedlichen Formen vor. Enthalten die Bestimmungen D 49 der Landesordnung vom 10. Mai 1627 und no. 84 und 85 des Codex Theresianus von 1758 keine bzw. noch keine référé-Bestimmungen, weisen die königlichen "Deklarationen“ und „Novellen“ Ferdinands III. von 1640 unter C c 5 sowie $\S 437$ der Allgemeinen Gerichtsordnung von 1781 und vor allem die $\S \S 24 f f$. des Josephinischen Gesetzbuches von 1786 eine Anfrageverpflichtung des Richters auf. Das Ende des référé leitet $\S 2$ des Patents von 1791 ein, indem es nur noch in den Fällen der Gesetzesanwendung zur Anfrage kommen sollte, bei denen der Richter besondere oder erhebliche Bedenken hatte. Das Westgalizische Gesetzbuch von 1797 und das ABGB von 1811 enthalten den référé législatif nicht mehr und verweisen den Richter auf „allgemeine und natürliche Rechtsgrundsätze".

\footnotetext{
${ }^{96} \mathrm{Zu}$ § 7: DitTrich, TADES, Allgemeines bürgerliches Gesetzbuch 9f.; WELLSPACHER, Naturrecht und ABGB $184 f$.
} 


\section{Schluss: Zusammenhänge zwischen référé législatif und Gesetzes- und Richterbild}

\section{Momentaufnahmen zum aufgeklärt-absolutistischen Gesetzes- und Richterbild hinter den référé législatif-Regelungen}

Gesetzgeberische Akte eines aufgeklärt-absolutistischen Monarchen in Form von Gesetzen, Ordonnanzen oder Edikten sind im Rahmen einer Vorlagepflicht von den Richtern als Herrscherbefehl aufzufassen, die den Willen des Gesetzgebers wiedergeben. Der Richter muss genau diesen Willen des Gesetzgebers bei der Gesetzesanwendung und -auslegung befolgen und - wenn notwendig - über einen référé législatif einholen. $\mathrm{Zu}$ dieser Rechtsdurchsetzungsfunktion gehört auch die Zentralisierungsfunktion des référé législatif: Die größtmögliche Rechtsvereinheitlichung gehört zur aufgeklärtabsolutistischen Staatsräson. Die österreichische Monarchie versucht, durch umfassende Kodifikationen das Recht zu vereinheitlichen, zu systematisieren und subsidiäre Rechtsquellen auszuschließen bzw. zurückzudrängen. Der référé législatif steht im Zusammenhang mit der Schaffung eines ius certum, gerade auch zur Durchsetzung des aufgeklärt absolutistischen Herrscherwillens. Mit dem zunehmenden Verständnis des "Gesetzes" als allgemeine Regel ${ }^{97}$ dient Gesetzgebung nicht mehr nur der Schaffung eines ius certum durch Fixierung und Besserung des vorgegebenen Rechts, sondern wird Instrument der bewussten Planung, Lenkung und

\footnotetext{
97 Vgl. zur Geschichte des Gesetzesbegriffs EBEL, Gesetzgebung 25f., 64f., 73f.; GAGNÉR, Studien 55ff., 107ff., 354 ff.; NEUMANN, Herrschaft 94f., 245f.; IMMEL, Typologie der Gesetzgebung 83f., 90f.; COING, Europäisches Privatrecht 1, 7ff., 31f., 252ff.
}

Kontrolle des Staatslebens ${ }^{98}$. Die restriktivsten référé-Bestimmungen im Josephinischen Gesetzbuch in Österreich stehen in unmittelbarer Verbindung mit der Verdrängung subsidiärer Rechtsquellen in Form des Gewohnheitsrechts oder des römischen Rechts.

Neben den Zielen der Gesetzesdurchsetzung und der Realisierung des Gesetzgeberwillens ist aber auch das Ansehen der Justiz und des Richtertums von maßgeblicher Bedeutung für die Begründung eines référé législatif. Jede Vorlagepflicht beinhaltet ein tiefes antijudizielles Misstrauen, Richter (und Advokaten) seien korrupt, nutzten ihr Fachwissen und gesetzliche Spielräume aus, um Verfahren zu verzögern und den Inhalt der Gesetze bewusst zu verfälschen. Diese Kontrollfunktion des référé législatif ist jedoch kein Selbstzweck, sondern wird instrumentalisiert im Kampf gegen ständisch beeinflusste Spruchkörper, welche nur langsam durch umfassende Justizreformen $\mathrm{zu}$ landesherrlichen Zentralbehörden umgeformt werden konnten. ${ }^{99}$ Dieser Aspekt wird in der bisherigen Literatur nicht aufgegriffen, 100 die die Geschichte des référé législatif zu sehr aus dem Blickwinkel der Gewaltenteilung erklären will. Dass dies nicht zu überzeugen vermag, zeigt schon die Inanspruchnahme Montesquieus für die Einführung und für die Abschaffung der richterlichen Vorlagepflicht: So sprach sich 1783 Rebeur in Preußen unter Berufung auf Montesquieus "De l'esprit des lois" gegen den référé aus, während die Deputierten der französischen Nationalversammlung ab 1790 die Einführung der richterlichen Vorlagepflicht gerade mit den Ausführun-

\footnotetext{
${ }^{98}$ Diese Zusammenhänge beschreiben außerdem ausführlich MAIER, Staats- und Verwaltungslehre 49ff., 72f., 153f.; TIGGES, Stellung des Richters 22ff.; DiESTELKAMP, Verhältnis von Gesetz- und Gewohnheitsrecht 27ff.; SCHULZE, Geschichte der neueren vorkonstitutionellen Gesetzgebung 161f., $201 \mathrm{f}$.

${ }^{99}$ MÜßIG, Recht und Justizhoheit, 218 ff.

100 Statt vieler MiERSCH, Référe législatif 48;. vgl. dazu auch SEIF, Rezension.
} 
gen Montesquieus begründeten. ${ }^{101}$ Wie ich bereits an anderer Stelle gezeigt habe, beruhen die Herausbildung des modernen Gesetzesbegriffes ${ }^{102}$ und die Entwicklung der Gewaltenteilung ${ }^{103}$ auf verschiedenen Entwicklungslinien. Zudem verkennt die Sekundärliteratur bisher die Interpretation von Montesquieus Kritik an der unmittelbaren Ausübung der Justizgewalt durch den Herrscher selbst oder durch Kommissare: Jeder unvermittelte Machtgebrauch beeinträchtigt die Freiheit. ${ }^{104}$ Die Mittelbarkeit der Herrschergewalt entspricht der Mäßigung der Staatsgewalt, wodurch politische Freiheit erst möglich wird. ${ }^{105}$ Dieser Gedanke der Mäßigung der Justizhoheit durch ordentliche Justizbeamte als selbständige Zwischengewalten ist im $\mathrm{Zu}-$ sammenhang zu sehen mit Montesquieus Ideal der monarchischen Mischverfassung und beruht daher gerade nicht auf einer konstitutionellen Gewaltentrennung. Die Vermittlung monarchischer Gewalt durch ständische Zwischengewalten (pouvoirs intermédiaires), die zusammen mit der Krone über das Staatswohl entscheiden, widerspricht dem Zentralisierungsstreben des josephinischen Absolutismus.

Folgt man der Annahme, dass monistische Fürstenstaatlichkeit nach der neueren Absolutismusforschung ${ }^{106}$ unerreichbar war und Herrschaft über die Justiz Herrschaft über das Recht ermög-

\footnotetext{
${ }^{101}$ MIERSCH, Référé législatif $137 f$.

102 SEIF, Recht und Gerechtigkeit.

103 SEIF, Montesquieu.

104 MonTEsQuieu, Geist der Gesetze VI, 5 (pag. 314).

${ }^{105}$ Politische Freiheit ist für Montesquieu die Mäßigung bei der Ausübung der souveränen Gewalt, unabhängig von der Regierungsform: „La liberté politique ne se trouve que dans les gouvernements modérés" (XI, 4 (pag. 395)). Die Freiheit ist damit für Montesquieu keineswegs direkt von der Regierungsform abhängig. Auch Republiken wie Venedig können tyrannisch sein. Zum ständischen Freiheitsbegriff Montesquieus vgl. BÖCKENFÖRDE, Gesetz und gesetzgebende Gewalt 30; SEIF, Montesquieu 152f.

106 Zusammenfassung des Forschungsstandes bei SEIF, Absolutismus, m.w.N.
}

lichte, steht die Geschichte von der Einführung und Abschaffung des référé législatif im Kontext der Auseinandersetzung um das monarchische Rechtsprechungsmonopol gegenüber dem feudalstaatlichen Gerichtspartikularismus. Mit dem référé konnten ständisch stark beeinflusste Spruchkörper zurückgedrängt werden. Mit den Veränderungen in der Ausbildung und bei der Bestellung der Richter durch die umfangreichen Justizreformen verlor die Funktion des référé im Kampf gegen ständisch stark beeinflusste Richterkollegien immer stärker an Bedeutung. Von der zunehmenden Unabhängigkeit der Justiz profitierten Monarch und Bürgertum. Wo der Monarch seine persönliche Verantwortung einbüßte und nur formell über die Gerichtsbarkeit selbst zu wachen hatte, kam den Bürgern die Aufhebung eines der wichtigsten ständischen Vorrechte gerade gelegen. ${ }^{107}$ Je stärker die Gerichte vom Zugriff des Souveräns bzw. des eigentlichen Machthabers entfernt sind und dieser u.a. aufgrund politischer, ideologischer oder sozialer Gründe ein tiefes Misstrauen gegenüber dem Richterstand entwickelt, desto intensiver ist der Wille des Souveräns, in den Prozess der Anwendung der Gesetzestexte auf den Einzelfall einzugreifen ${ }^{108}$ und desto restriktiver ist die Ausprägung des référé législatif für den Richter.

Rezipiert wird dagegen Montesquieus Herrschaft der Gesetze. Der Gesetzesstaat steht entsprechend der vorrangigen Herrschaftsfunktion der Gesetzgebung - für den Ausbau der Monarchie in der Donaumonarchie. Beginnend mit dem Codex Theresianus nahm die österreichische Krone Gesetzgebungsvorhaben in An-

${ }^{107}$ LÖHNIG, Zur Stellung des Richters 430f.

108 Vgl. NeumanN, Herrschaft 262ff.; Tigges, Stellung des Richters 15ff., 30ff., 53f.; FRIEDRICH, Verfassungsstaat 115ff.; RÜCKERT, Autonomie des Rechts 38f.; OGOREK, Politisches Selbstverständnis 72f.; Coing, Europäisches Privatrecht 31f., 252ff.; WEBER, Schöppenstuhl und Landesherr 263f., 268f.; SELLERT, Unabhängigkeit des Richters 443-451; KAUFMANN, Urteil 603-609. 
griff, deren Ziel die systematisch angelegte Regelung zusammengehöriger Rechtsmaterien in umfassenden Kodifikationen war. Dieses Streben nach Rechtssicherheit verbindet die Idee des Rechts untrennbar mit dem rationalzweckorientierten und ordnungsgemäß publizierten Gesetz. Ein derartiger Primat der Gesetzgebung führt zu einem neuen Verständnis der Richterentscheidung: Der Rechtspruch eines an das Gesetz gebundenen Beamten unterscheidet sich jetzt nicht mehr nur formalinstitutionell, sondern auch inhaltlich vom Machtspruch des in seiner Machtfülle (plenitudo potestatis) allein Gott verantwortlichen Monarchen. Der inhaltliche Gegensatz zwischen gesetzesgemäßer Richterentscheidung und politischer Kabinettsentscheidung war aus der Taufe gehoben. Der vernunftbestimmte Normzweck gilt für Justizsachen, der politische Wille des Monarchen ist für Regierungssachen maßgeblich. Mit dem Vorrang des vernunftbestimmten Normzwecks vor dem Herrscherwillen im aufgeklärten Gesetzesstaat gerät die Richtervorlage in die Nähe der Kabinettsjustiz. Die Anordnungen des monarchischen Gesetzgebers nach einer richterlichen Anfrage greifen nämlich rückwirkend in den anhängigen Einzelfall ein.

Durch den Vorrang des vernunftbestimmten Normzwecks vor dem monarchischen Herrscherwillen darf der Monarch als Träger ungeteilter Staatsgewalt und oberster Gerichtsherr von seiner Gerichtsherrschaft keinen Gebrauch mehr machen. Dieser mit den ständischen Interessen in der Justizverwaltung einhergehende Kern für die Unabhängigkeit der richterlichen Entscheidung gegenüber monarchischer Einmischung via référé beruht weder auf einer konstitutionellen Gewaltentrennung noch beinhaltet sie ein individuelles Justizgrundrecht. Vielmehr erscheint sie als Ergebnis der Präzisierung des Gesetzesbegriffes in der Auseinandersetzung um den référé législatif als abstrakte Regel, ${ }^{109}$ die keine Einzelfälle oder -personen erwähnt, in die Zukunft wirkt und nur abstrakt angewendet werden kann. Die Anordungen des Gesetzgebers nach einer richterlichen Anfrage dagegen greifen rückwirkend in den anhängigen Einzelfall ein. Der so verdeutlichte Allgemeinheitscharakter des Gesetzes hat Auswirkungen auf die richterliche Tätigkeit, da nun ausschließlich dem Richter die Anwendung des Gesetzes auf den Einzelfall überlassen bleibt und er auf die „natürlichen Rechtsgrundsätze" verwiesen wird. Insoweit war der référé législatif Katalysator für die richterliche Unabhängigkeit.

\section{Auseinandersetzung mit der rechtstheoretischen Forschungs- kontroverse Lukas/Hatschek}

Die Richtigkeit des hier artikulierten Zusammenhanges zwischen référé législatif und Gesetzes- und Richterbild ist nicht auf die rechtstheoretische Kontroverse zwischen den JellinekSchülern Josef Lukas und Julius Hatschek beschränkt. ${ }^{110}$ Während für Lukas die Naturrechtskodifikationen im Licht der eigenen Lückenhaftigkeit ${ }^{111}$ das Naturrecht als subsidiäre Rechtsquelle anerkennen (Art. 4 des Code Napoléon; §§ 18, 19 WGGB; §§ 6, 7 ABGB) ${ }^{112}$ und damit den Richter jenseits der Vorlagepflicht noch als Rechtsanwender sehen, ${ }^{113}$ wertet Hatschek aufgrund seines benthamschen Verständnisses den Naturrechtsverweis als Bezug

${ }^{109}$ Zur Allgemeinverbindlichkeit des Gesetzes NeumanN, Herrschaft 245ff, Anm. 102; ScHulze, Geschichte 204f. sowie EBEL, Geschichte 91ff.; SCHMITT, Verfassungslehre 141f.

${ }^{110}$ Dazu folge den Streitschriften LUKAS, Zur Lehre 418ff.; HATSCHEK, Bentham und die Geschlossenheit 442ff; LUKAS, Benthams Einfluss 67ff., 465ff.; HATSCHEK, Bentham und die Geschlossenheit 458ff.

${ }^{111}$ LUKAS, Benthams Einfluss 67.

112 LUKAS, Benthams Einfluss 74, 82f., $89 \mathrm{f}$.

${ }^{113}$ LUKAS, Zur Lehre 423; DERS., Benthams Einfluss 74, 109. 
auf die „Natur der Sache“. Ein solcher Hinweis auf das freie richterliche Ermessen ${ }^{114}$ bestätigt ihm die Geschlossenheit der Kodifikation. ${ }^{115}$ Recht haben beide darin, dass mit der Anerkennung der Rechtsquellenqualität des natürlichen Rechts (ABGB) und des natürlichen Rechts mit dem Gewohnheitsrecht (Code Civil) die Richter unbehelligt von einer Vorlagepflicht letztlich Rechtsanwender blieben: in einer am Gesetz orientierten rechtsfortbildenden Auslegung kann der Richter im Falle des Schweigens des Gesetzes auf natürliches Recht bzw. Gewohnheitsrecht als „unversiegbarer Quell“116 an Stelle des référé législatif zurückgreifen, ohne dass die rechtstheoretische Einordung des Gesetzbuches pro oder contra Bentham eine Rolle spielen würde.

Lukas übersieht jedoch, dass das österreichische Patent vom 22. Februar 1791 außerhalb Galiziens die Abschaffung bzw. starke Eingrenzung des référé législatif auch ohne Verweis auf das Naturrecht ermöglichte. ${ }^{117}$ Und Hatschek isoliert den Vortrag der österreichischen Hofkommission vom 29. Januar 1808, in dem es heißt: „Wenn der Bürger aus dem Codex den ganzen Umfang seines Privatrechtes soll erkennen können, so ist die IV. wesentliche Eigenschaft die Vollständigkeit. Der Codex darf nicht nur keinen Zweig der Rechtsgeschäfte übergehen, sondern er muss auch für jeden Rechtszweig so erschöpfende Vorschriften enthalten, dass der Richter und Rechtsgelehrte jeden möglichen Rechtsfall daraus zu entscheiden fähig sei.“118 Schon bei Zeil-

\footnotetext{
114 HATSCHEK, Bentham und die Geschlossenheit 452.

${ }^{115}$ HATSCHEK, Bentham und die Geschlossenheit 456. Vgl. auch ibid. (460) mit dem Hinweis auf den Code Napoléon, der den Richter nur auf das Gesetz und als Ergänzung auf die Billigkeit und das freie richterliche Ermessen verweist.

116 In Österreich ZEILLER, Commentar 1，21，66 (MiERSCH, Référé législatif 170, 173).

117 Dies bemerkt auch SPIEgEL, Référé législatif 116.

${ }^{118}$ HATSCHEK, Bentham und die Geschlossenheit 444; dazu auch OFNER, Ur-Entwurf 2, 469.
}

ler deutete sich eine geänderte Auffassung über die Funktion und den Begriff des Gesetzes an. Dieses solle die Bürger „im Voraus belehren“. Die Betonung der Allgemeinheit des Gesetzes und die Ausgrenzung der gesetzgeberischen von der richterlichen Tätigkeit beeinflussten den Substanzverlust des référé.

Zudem erscheint die Forschungskontroverse Lukas/Hatschek auf theoretische Erwägungen verengt. Die Aufhebung des référé législatif hatte doch wohl auch rein praktische Gründe: So bekennt das den référé législatif aufhebende Februarpatent 1791 ausdrücklich, die gewünschten Ziele nicht erreicht zu haben, ${ }^{119}$ zumal die Bejahung einer Vorlagenotwendigkeit allein von der subjektiven Einschätzung des Richters abhängig war, der ohne jeden Zweifel auch aus Vorsicht oder aus Bequemlichkeit anfragen konnte. Daher mögen die Überlastung der mit der Beantwortung betrauten Stellen, die Kostspieligkeit der Anfragen und die zunehmenden Verzögerungen der anhängigen Rechtsstreitigkeiten die wahren praktischen Gründe für die Aufhebung des référé sein.

Im Ergebnis jedenfalls haben Aufstieg und Fall des référé législatif der richterlichen Unabhängigkeit in der österreichischen Verfassungsgeschichte den Weg bereitet.

\footnotetext{
119 Patent in Österreich vom 22. 2. 1791 und Kabinettsorder in Preußen vom 8. 3. 1798
} 


\section{Korrespondenz:}

Dr. Ulrike Müßig

Universität Passau

Lehrstuhl für Bürgerliches Recht sowie Deutsche

und Europäische Rechtsgeschichte

Innstraße 39 (Juridicum)

94032 Passau

ulrike.muessig@uni-passau.de

\section{Abkürzungen:}

Cap. Capitel

no. numero

Nov. Novelle

\section{Literatur:}

Hans-Jürgen BECKER, Kommentier- und Auslegungsverbote, in: $\mathrm{HRG}^{1}$, Bd. 2 (Berlin 1978) 963-974.

Hans-Wolfgang BergerHAUSEN, Die „Verneuerte Landesordnung" in Böhmen 1627: ein Grunddokument des habsburgischen Absolutismus, in: HZ 272 (2001) 327-351.

Friedrich BERGMANN, Das Verbot der rückwirkenden Kraft neuer Gesetze im Privatrechte, 1. und 2. Buch (Hannover 1818).

Hartwig BRANDT, Landesständische Repräsentation im deutschen Vormärz (Berlin 1968).

Pio CARONI, Kodifikation, in: $\mathrm{HRG}^{1}$, Bd. 2 (Berlin 1978) 907-922.

Helmut CoING, Europäisches Privatrecht, 2 Bde. (München 1989).

Hermann CONRAD, Deutsche Rechtsgeschichte, Bd. 2: Neuzeit-1806 (Karlsruhe 1966).

Hermann CONRAD, Richter und Gesetz im Übergang vom Absolutismus zum Verfassungsstaat (Graz 1971).

Bernhard DiestelKAMP, Das Verhältnis von Gesetzund Gewohnheitsrecht im 16. Jahrhundert - aufgezeigt am Beispiel der Oberhessischen Erbgewohnheiten von 1572, in: Karl KROESCHELl (Hg.), Festschrift für Hans Thieme zum 80. Geburtstag (Sigmaringen 1977) 1-33.

Robert DITTRICH, Helmuth TADES, Das Allgemeine Bürgerliche Gesetzbuch samt den einschlägigen Gesetzen und Verordnungen, verweisenden und erläuternden Anmerkungen, Literaturangaben und einer Übersicht über die Rechtsprechung der Gerichte, insbesondere des Obersten Gerichtshofes (Wien ${ }^{33} 1989$ ).
Bernadette Droste-LeHNEN, Die authentische Interpretation. Dogmengeschichtliche Entwicklung und aktuelle Bedeutung (Baden-Baden 1990).

Wilhelm EBEL, Geschichte der Gesetzgebung in Deutschland (Göttingen 1988).

Iring FETSCHER, Rousseaus politische Philosophie. Zur Geschichte des demokratischen Freiheitsbegriffes (Baden-Baden ${ }^{3}$ 1978).

Andreas FIJAL, Winfried ELLERBROCK, Das Österreichische ABGB vom 1. Juni 1811 - ein Jubiläum besonderer Art, in: Juristische Schulung 28/Nr. 7 (1988) 519-523.

Carl J. FRIEDRICH, Der Verfassungsstaat der Neuzeit (Berlin 1953).

Sten GAGNÉR, Studien zur Ideengeschichte der Gesetzgebung (Uppsala 1960).

François GENY, Méthode d'interprétation et sources en droit privé positif, Bd. 1 (Paris 1932).

Wolfgang GITTER, Die Methode der richterlichen Gesetzesauslegung als staatsrechtliches Problem, Versuch einer Darstellung der Methodenlehre in ihrer Beziehung zum Staatsrecht (Tübingen 1960).

Philipp Harras von HARRASOWSKY, Geschichte der Codification des österreichischen Civilrechts (Frankfurt am Main 1868).

Philipp Harras von HARRASOWSKY, Der Codex Theresianus und seine Umarbeitungen, 5 Bde. (Wien 1883-1886).

Julius HATSCHEK, Bentham und die Geschlossenheit des Rechtssystems, in: Archiv des Öffentlichen Rechts 24 (1909) 442-458.

Hans Hattenhauer, Geschichte des deutschen Beamtentums, (Berlin ${ }^{21993) . ~}$

Yves-Louis HufteAu, Le référé législatif et les pouvoirs du juge dans le silence de la loi (Paris 1965).

Max IMboden, Rousseau und die Demokratie (Tübingen 1963).

Gerhard IMMEL, Typologie der Gesetzgebung des Privatrechts und Prozeßrechts, in: Helmut COING (Hg.), Handbuch der Quellen und Literatur der neueren europäischen Privatrechtsgeschichte, Bd. 2/2 (München 1976).

Ekkehard KAUFMANN, Urteil (rechtlich), in: HRG ${ }^{1}$, Bd. 5 (Berlin 1993) 603-609.

Karl LOEWENSTEIN, Verfassungslehre (Tübingen 1959).

Martin LÖHNIG, Zur Stellung des Richters im Frühkonstitutionalismus, in: Tijdschrift voor Rechtsgeschiedenis 80 (2012) 437-441.

Max LANDMANN, Der Souveränitätsbegriff bei den französischen Theoretikern von Jean Bodin bis auf Jean Jacques Rousseau (Leipzig 1896). 
Josef LUKAS, Zur Lehre vom Willen des Gesetzgebers, in: Festgabe für Paul Laband (Tübingen 1908) 397427.

Josef LUKAS, Benthams Einfluss auf die Geschlossenheit der Kodifikation, in: Archiv des Öffentlichen Rechts 26 (1910) 67ff.

Hans MAIER, Die Ältere Staats- und Verwaltungslehre (München 21980).

Philippe-Antoine MERLIN, Répertoire universel et raisonné de jurisprudence, $\mathrm{Bd} .10$ (Paris ${ }^{3} 1809$ ).

Matthias MiERSCH, Der sogenannte référé législatif, in: Fundamenta Juridica (= Beiträge zur rechtswissenschaftlichen Grundlagenforschung 36, BadenBaden 2000).

Heinz Mohnhaupt, Potestas legislatoria und Gesetzesbegriff im Ancien Régime, in: Ius Commune 4 (1972) 188-239.

Charles-Louis Montesquieu, Vom Geist der Gesetze (ND Stuttgart 1994).

Ulrike MÜßIG, Recht und Justizhoheit, Der gesetzliche Richter im historischen Vergleich von der Kanonistik bis zur Europäischen Menschenrechtskonvention, unter besonderer Berücksichtigung der Rechtsentwicklung in Deutschland, England und Frankreich (Berlin 22009).

Franz NeumanN, Die Herrschaft des Gesetzes (Frankfurt am Main 1980).

Regina OGoreK, De l'Esprit des légendes, in: Rechtshistorisches Journal 2 (1983) 277-296.

Regina OGOREK, Zum Politischen Selbstverständnis der Rechtsprechung am Vorabend des bürgerlichen Zeitalters - eine Fallstudie, in: Ius Commune 10 (1983) 69-95.

Julius OFNER, Der Ur-Entwurf und die Berathungsprotokolle des Österreichischen Allgemeinen Bürgerlichen Gesetzbuches, Bd. 1 (Wien 1889).

Carl Eduard Oтто, Bruno Schilling, Carl Friedrich Ferdinand SINTENIS, Corpus Iuris Civilis, 7. Bd (Leipzig 1831-1839, ND Aalen 1985).

H. Peter, Englisches Recht, in: HRG ${ }^{1}$, Bd. 1 (Berlin 1971) 921-939.

Frank R. PfETSCH, Verfassungspolitik der Nachkriegszeit (Darmstadt 1985).

Jean Jacques RousseAU, Staat und Gesellschaft (München 1959).

Joachim RÜCKERT, Autonomie des Rechts in rechtshistorischer Perspektive (Hannover 1988).

Helmut QUARITSCH, Souveränität, in: $\mathrm{HRG}^{1}$, Bd. 4 (Berlin 1990) 1714-1725.

Carl SCHMITT, Verfassungslehre (Berlin 1983).

Clausdieter SсHOTт, „Rechtsgrundsätze“ und Gesetzeskorrektur (Berlin 1975).
Reiner SCHULZE, Geschichte der neueren vorkonstitutionellen Gesetzgebung, in: ZRG GA 98 (1981) 157-253.

Wolfgang SELLERT, Unabhängigkeit des Richters (der Justiz), in: HRG ${ }^{1}$, Bd. 5 (Berlin 1998) 443-451.

Thomas SIMON, Gesetzgebungsstaat und die „Bindung des Richters an das Gesetz" (= Antrittsvorlesungen der Rechtswissenschaftlichen Fakultät der Universität Wien 1, Wien 2008).

Ulrike SEIF (seit 2006 MÜßIG), Der mißverstandene Montesquieu. Gewaltenbalance, nicht Gewaltentrennung, in: ZNR 22 (2000) 149-166.

Ulrike SEIF (seit 2006 MÜßIG), Rezension von: Matthias Miersch, Der sogenannte référé legislatif, in: ZRG GA 119 (2002) 720-722.

Ulrike SEIF (seit 2006 MÜßIG), Recht und Gerechtigkeit. Die Garantie des gesetzlichen Richters und die Gewaltenteilungskonzeptionen des 17.-19. Jahrhunderts, in: Der Staat 42 (2003) 110-140.

Ulrike SEIF (seit 2006 MÜßIG), Absolutismus, in: $\mathrm{HRG}^{2}$, Bd. 1 (Berlin 2004) 30-38.

Ludwig SPIEGEL, Der référé législatif oder die Anfrage bei Hof, in: Gesetz und Recht, Vorträge und Aufsätze zur Rechtsquellentheorie (München-Leipzig 1913).

Otto Sтовве, Geschichte der deutschen Rechtsquellen, in: Geschichte des deutschen Rechts, Bd. 2 (Braunschweig 1864).

Michael STOLLEIS, Geschichte des öffentlichen Rechts in Deutschland, Bd. 2 (München 1992).

Hans Tigges, Die Stellung des Richters im modernen Staat (Berlin 1935).

Thilo Vogel, Zur Praxis und Theorie der richterlichen Bindung an das Gesetz im gewaltenteilenden Staat (Berlin 1969).

Friedrich WALTER, Österreichische Verfassungs- und Verwaltungsgeschichte von 1500-1955 (WienKöln-Graz 1972).

Hellmuth von WEBER, Schöppenstuhl und Landesherr, in: Festschrift für Richard Thoma (Tübingen 1950).

Moritz WELLSPACHER, Das Naturrecht und das ABGB, in: Festschrift zur Jahrhundertfeier des Allgemeinen Bürgerlichen Gesetzbuches, 1. Teil (Wien 1911) 175-207.

Franz WIEACKER, Privatrechtsgeschichte der Neuzeit unter besonderer Berücksichtigung der deutschen Entwicklung (Göttingen 21967).

Franz von ZeILler, Commentar über das allgemeine bürgerliche Gesetzbuch für die gesammten Erbländer der Österreichischen Monarchie, Bd. 1 (Wien-Triest 1811). 